\title{
Contribution of Bottom-Up and Top-Down Motion Processes to Perceived Position
}

\author{
David Whitney \\ University of California, Davis
}

\begin{abstract}
Perceived position depends on many factors, including motion present in a visual scene. Convincing evidence shows that high-level motion perception-which is driven by top-down processes such as attentional tracking or inferred motion - can influence the perceived position of an object. Is high-level motion sufficient to influence perceived position, and is attention to or awareness of motion direction necessary to displace objects' perceived positions? Consistent with previous reports, the first experiment revealed that the perception of motion, even when no physical motion was present, was sufficient to shift perceived position. A second experiment showed that when subjects were unable to identify the direction of a physically present motion stimulus, the apparent locations of other objects were still influenced. Thus, motion influences perceived position by at least two distinct processes. The first involves a passive, preattentive mechanism that does not depend on perceptual awareness; the second, a top-down process that depends on the perceptual awareness of motion direction. Each contributes to perceived position, but independently of the other.
\end{abstract}

Keywords: vision, localization, crowding, transformational apparent motion, motion aftereffect

The perceived position of an object is influenced by the visual motion that is present in a scene. For example, moving objects can appear shifted in the direction of their motion (De Valois \& De Valois, 1991; Durant \& Johnston, 2004; Edwards \& Badcock, 2003; Fang \& He, 2004; McGraw, Whitaker, Skillen, \& Chung, 2002; Mussap \& Prins, 2002; Nishida \& Johnston, 1999; Ramachandran \& Anstis, 1990; Snowden, 1998; Whitaker, McGraw, \& Pearson, 1999), and stationary flashed objects in one location can be shifted by motion in another location in the visual field (Whitney \& Cavanagh, 2000, 2003). In addition to these influences of motion on perceived position of static objects, there are potentially related phenomena revealing an interaction between visual motion and perceived location. These include the flash-lag effect (for reviews, see Krekelberg \& Lappe, 2001; Nijhawan, 2002; Whitney, 2002), representational momentum (for reviews, see Thornton \& Hubbard, 2002), and several other phenomenologically similar effects (Thornton, 2002; Thorson, Lange, \& Biederman-Thorson, 1969; for a review, see Whitney, 2002). Most of the psychophysical studies on these effects have used luminance-defined (firstorder) motion, suggesting that motion may influence position assignment at a relatively early stage, such as primary visual cortex, motion area MT+ (McGraw, Walsh, \& Barrett, 2004), or perhaps an interaction between these two areas (Nishida \&

Parts of this work were presented at the Vision Sciences Society in Sarasota, FL, May 2005, and part of Experiment 2 was reported in brief form in Current Biology. This work was supported by funding from the University of California, Davis. Thanks go to David Bressler for help collecting data.

Correspondence concerning this article should be addressed to David Whitney, Center for Mind and Brain and Department of Psychology, University of California, One Shields Avenue, Davis, CA 95616. E-mail: dwhitney@ucdavis.edu
Johnston, 1999; Whitney \& Cavanagh, 2000). Mounting physiological evidence supports this perspective; the retinotopic specificity of neurons in the retina and primary visual cortex are modulated by motion (Berry, Brivanlou, Jordan, \& Meister, 1999; Fu, Shen, \& Dan, 2001; Fu, Shen, Gao, \& Dan, 2004; Whitney et al., 2003).

The psychophysical and physiological experiments above have provided converging evidence that low-level motion (analyzed by passive, bottom-up processes) influences coded position at, perhaps, a number of levels in the visual hierarchy. However, the similarity between the stimuli used in the psychophysical and the physiological experiments makes it easy to overlook the possibility that there could be other, higher level contributions of motion to position coding.

For example, a few recent studies have demonstrated that strict low-level motion is not necessary to shift the apparent location of an object. Watanabe and colleagues (K. Watanabe, Nijhawan, \& Shimojo, 2002; K. Watanabe, Sato, \& Shimojo, 2003) found that when an object travels behind an occluder so that only a single pixel-thin line of the object is visible at one time (a version of anorthoscopic motion), there is an impression that the object moves horizontally behind the occluder; of importance, however, there is no actual horizontal motion in the image. Subjects perceive the horizontal motion of the image by inferring it from the information provided within the single pixel-wide aperture. This demonstration is nice because it shows that the physical or low-level luminance defined motion in a scene is not necessarily what determines the perceived position of an object. Inferred, high-level motion perception also contributes to perceived location.

In another study, Shim and Cavanagh (2004) found that an ambiguous apparent motion sequence could shift the perceived locations of nearby objects depending on the direction of motion that subjects perceived in the apparent motion display. The display consisted of a bistable quartet (Ramachandran \& Anstis, 1983; 
Ternus, 1938); when asked to attend to one direction of motion (either the vertical or the horizontal), observers were able to modulate which direction was dominant (Suzuki \& Peterson, 2000). When subjects attended to one direction of motion, there was a significant shift in the perceived position of another irrelevant object that was briefly presented. There was little or no illusory shift in the unattended direction.

Together, these recent studies convincingly show that attention to, or inference of, motion can alter humans' perception of object position, but they do not determine whether this high-level, topdown motion perception mediates all of the motion-induced position displacement illusions mentioned above. Here we test whether a single high-level motion representation or inference can explain most or all of the motion-induced position displacement illusions.

In all previous demonstrations of motion's influence on perceived position, the perceived shift has always been accompanied (or preceded, in the case of the motion aftereffect) by a visible and salient motion signal. Observers can easily scrutinize the motionthey can attentionally track, or at least perceive through inference, the moving features. In other words, what is similar about all of the stimuli is that observers are always aware of the motion direction, and the illusory position shift is always consistent with this awareness. In the case of the position shifts that accompany the motion aftereffect (Fang \& He, 2004; McGraw et al., 2002; Nishida \& Johnston, 1999; Snowden, 1998; Whitaker et al., 1999; Whitney \& Cavanagh, 2003), for example, observers can attend to (Chaudhuri, 1990; Mather, Verstraten, \& Anstis, 1998; Mukai \& Watanabe, 2001) or attentively track (Cavanagh, 1992; Culham, Verstraten, Ashida, \& Cavanagh, 2000; Seiffert, Somers, Dale, \& Tootell, 2003) the moving adaptation stimulus. Even if the test pattern does not display any perceived motion (McGraw et al., 2002; Whitney \& Cavanagh, 2003), the moving adaptation stimulus is clearly visible, and it could be the visibility of the adaptation stimulus that ultimately shifts the perceived position of the test stimulus.

In this study, we tested whether there are contributions of both bottom-up and top-down motion processes to the perception of position. One of the goals of these experiments was to address whether the awareness of motion direction is necessary to influence the perceived position of an object. We found that despite the inability of observers to attentionally resolve or report the direction of motion, an object can still appear shifted. The results show that visual motion influences coded location whether or not subjects are aware of the motion, suggesting that both bottom-up and top-down mechanisms contribute to perceived position.

\section{Experiment 1A: High-Level Motion Perception Influences Perceived Position}

Ultimately, the question in this study is whether motion influences perceived position only when subjects are aware of the motion. The first step in answering this question, however, is to confirm that awareness of motion is sufficient to influence perceived position. The key to addressing this question is to construct a display in which there is awareness of motion but no actual motion. As discussed above, several authors have done this; even when the motion in a scene is ambiguous, subjects often perceive unambiguous motion, and this perception influences apparent location (Shim \& Cavanagh, 2004; K. Watanabe et al., 2002, 2003). These studies used high-level motion displays that were contingent on either inferential processes (e.g., solving the aperture problem across multiple elements to determine object motion through a narrow window; K. Watanabe et al., 2002) or attentional processes (e.g., attending to one direction of motion over another; Shim \& Cavanagh, 2004). However, in these examples of high-level or top-down motion perception, there are physically moving elements, or at least features that change position over time. Thus, it is hard to distinguish whether the percept (or awareness) of the motion is alone necessary (e.g., attention might serve to filter out irrelevant directions of motion that produce a bias in the direction of the position shift). Using a display that elicits the percept of motion but contains no moving elements-not even ambiguous motion-will demonstrate that awareness of motion is sufficient to influence perceived position.

In the first experiment, we needed a display that elicits a strong impression of motion but contains no physical movement; moreover, this display should not contain any elements that change position over time or undergo ambiguous motion. To accomplish these restrictive goals, we used an illusion called transformational apparent motion (Tse \& Logothetis, 2002; cf. Chatterjee, Freyd, \& Shiffrar, 1996; Farrell \& Shepard, 1981; Ramachandran, Armel, Foster, \& Stoddard, 1998; Zanker, Quenzer, \& Fahle, 2001). Transformational apparent motion is a two-frame sequence in which an object is perceived to smoothly extend its boundaries from one position to another (Figure 1). For example, when the stimulus in Figure 1A is presented, the midline across the $\mathrm{H}$ stimulus appears to grow out from one side of the figure to the other (arrows in Figure 1B). The direction of the growing motion depends on where the cues (the small rectangular bumps in Frame 1 of Figure 1A) are located. Transformational apparent motion has been demonstrated in many different ways (Tse \& Logothetis, 2002), but for our purposes, the most important feature of transformational apparent motion is that it does not involve a change in the position of an object over time. The first frame of the sequence is always visible. The second frame is simply abruptly presented. There are no low-level or even ambiguous motion signals. The impression of motion is produced solely by the structure of the visible surfaces present in the first frame, and thus this serves as a good example of motion awareness without any actual motion.

Using a transformational apparent motion display (Figure 1A), we investigated whether the impression of motion can influence the apparent locations of nearby static objects (Figure 1C). If the illusory motion does influence the perceived position of an object, this would strongly support previous studies (Shim \& Cavanagh, 2004; K. Watanabe et al., 2002, 2003) that showed the influence of high-level motion on perceived position and, more important, would extend these previous findings to show that awareness of motion (without any physical motion) is sufficient to shift apparent position.

\section{Method}

Four subjects participated in the experiment, one of whom was the author. Three additional experienced psychophysical observers were naive as to the purpose of the experiment. Each had normal or corrected-tonormal vision. Subjects were seated in a darkened experimental booth with a chin rest $28 \mathrm{~cm}$ from a Sony G520 Multiscan CRT $(1,024 \times 768$ resolution) monitor, which had a refresh rate of $100 \mathrm{~Hz}$.

Stimuli were similar to that shown in Figure 1 and consisted of a two-frame sequence. There were two transformational apparent motion 


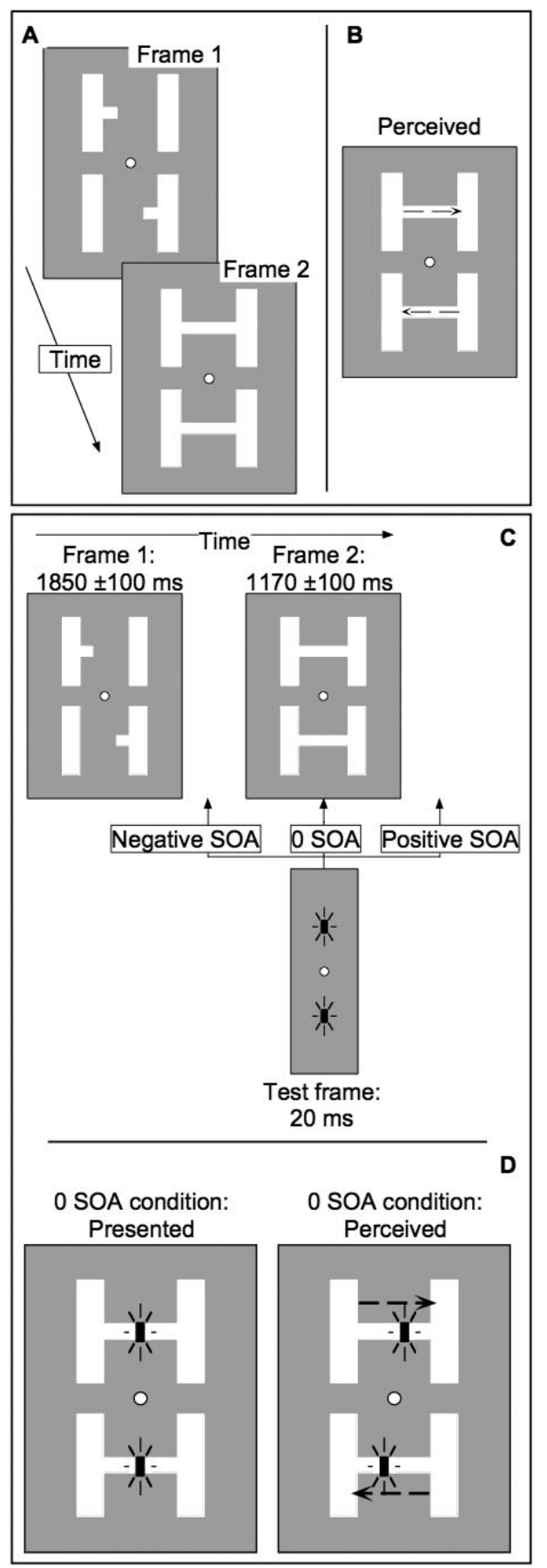

Figure 1. Transformational apparent motion illusion and stimuli used in the first experiment. A: Two frames are presented in a sequence. The stimulus consisted of an $\mathrm{H}$-shaped figure with part of the horizontal midline removed (Frame 1). There was a small rectangular bump (cue) on one of the vertical stalks of the $\mathrm{H}$ stimulus. The horizontal midline was presented all at once in Frame 2. B: Although the midline was physically stationary and did not change position over time, it appeared to move- to grow from one side to the other side of the $\mathrm{H}$ stimulus (arrows), depending on the location of the cue in Frame 1. The direction of the illusory transformational apparent motion is dictated by the location of the cue. C: Stimuli used in the first experiment. The first frame of a transformational apparent motion display was presented for $\sim 1,850 \mathrm{~ms}$. The second frame displays that were stacked vertically. Each display consisted of an $\mathrm{H}$-shaped stimulus. In the first frame of the sequence (Figure 1C, left panel), the horizontal midline of the two $\mathrm{H}$ stimuli was missing. In the second frame (Figure 1C, right panel), the horizontal midline was presented in both $\mathrm{H}$ stimuli simultaneously. Notice that in Figure 1C, in the first frame, there is a rectangular "bump" (cue) located on one of the vertical stalks of each $\mathrm{H}$ stimulus. This cue determines the direction of the illusory transformational apparent motion (Tse \& Logothetis, 2002), which was always in opposite directions in the two $\mathrm{H}$ stimuli (see arrows in Figure 1D). If the top $\mathrm{H}$ contained illusory rightward motion, the bottom $\mathrm{H}$ contained illusory leftward motion, and vice versa. The vertical stalks of the $\mathrm{H}$ stimulus were $7.7^{\circ} \times 1.6^{\circ}$, and the stalks were separated by $9.2^{\circ}$ (center to center, so the horizontal midline, presented in the second frame, filled that $9.2^{\circ}$ separation). The cue height and width were $0.8^{\circ} \times 0.9^{\circ}$, respectively. The vertical separation between the two $\mathrm{H}$ stimuli was $15^{\circ}$, and the fixation bull's-eye was centered between them. The background luminance was $29.5 \mathrm{~cd} / \mathrm{m}^{2}$. The luminance of the $\mathrm{H}$ stimuli was $90 \mathrm{~cd} / \mathrm{m}^{2}$ (50.6\% Michelson contrast).

On each trial, the first frame of the transformational apparent motion sequence was presented for $1,850 \pm 100 \mathrm{~ms}$, followed by the second frame of the sequence, which was visible for the remainder of the trial $(\sim 1,170$ $\mathrm{ms}$ ). The duration of the first frame was randomized within a 200-ms window to reduce predictability. At various times before or after the presentation of the second frame, two flashed objects were presented (Figure 1C). The two flashed targets were superimposed on the midline between the stalks of the $\mathrm{H}$ stimuli and were therefore vertically separated by $15^{\circ}$ (center to center). The two flashes were each $0.4^{\circ} \times 0.8^{\circ}$, were 1.55 $\mathrm{cd} / \mathrm{m}^{2}$, and were presented for $20 \mathrm{~ms}$ (96.6\% Michelson contrast). The flashes could be presented at any one of seven stimulus onset asynchronies (SOAs) from $250 \mathrm{~ms}$ before to $50 \mathrm{~ms}$ after the second frame. The flashes could be vertically aligned (as shown in Figure 1A) or could be misaligned by one of five possible equally spaced misalignments, spanning a $1.6^{\circ}$ range (symmetric about the horizontal midpoint between the flashes; each misalignment was equally probable). The SOA and flash misalignment were randomized on each trial, and in a method-of-constant-stimuli task, subjects were asked to judge the relative alignment of the two flashed objects (whether the top flash was to the left or the right of the bottom flash). Immediately after subjects responded, another trial was presented. The location of the cue (on the left or right of the display) in the two $\mathrm{H}$ stimuli was randomly determined on each trial, so the direction of transformational motion was also random (though always in opposite directions on the top and bottom $\mathrm{H}$ displays). Because the data for the two directions of transformational apparent motion were comparable, the data were flipped and merged.

In each experimental session, there were seven possible SOAs, six possible flash alignments at each SOA (i.e., aligned, or misaligned by one of five values), and 10 repeated trials for each of these six flash alignments, for a total of 420 trials per session. For each of the seven SOA conditions, a psychometric function was fit to the data from the logistic function $f(x)=$ $[1 /(1+\exp (-a(x-b)))]$, where $b$ estimates the physical misalignment between the flashes that creates an apparent alignment (the point of subjective equality [PSE]; Finney, 1971; McKee, Klein, \& Teller, 1985)

was presented for $\sim 1,170 \mathrm{~ms}$. Two test objects were flashed on the screen, superimposed on the location of the horizontal midline of the $\mathrm{H}$-shaped stimuli (labeled "Test frame"). The flashes could be presented at any one of 10 stimulus onset asynchronies (SOAs), either before, simultaneous with, or after the presentation of Frame 2. D: When the test flashes were presented at 0 SOA (i.e., simultaneous with Frame 2), the flashes appeared misaligned-shifted in position following the direction of the illusory transformational apparent motion. The direction of the illusory motion is indicated by the arrows. 
and $a$ is the slope of the function. For example, when the flashes were physically aligned, they appeared shifted in a direction consistent with that of the illusory transformational apparent motion (Figure 1D, right panel); in order to offset this apparent misalignment, the flashes had to be physically misaligned in a direction opposite that of the illusory motion. Each subject participated in three separate sessions $(420 \times 3$ sessions $=1,260$ trials), yielding three psychometric functions for each of the seven SOAs. The three psychometric functions for each SOA were averaged to yield a mean PSE, and standard errors were calculated on the basis of this.

\section{Results}

Consistent with work by Tse and Logothetis (2002), subjects reported perceiving strong transformational apparent motion in the displays in a direction dependent on where the cue was located. Figure 2A shows the perceived misalignment between the flashed targets produced by the transformational apparent motion for one representative subject in the $150-\mathrm{ms}$ SOA condition. If the flashed targets were perceived veridically, the PSE would have fallen at zero. The fact that the PSE was $\sim 21 \mathrm{~min}$ of arc indicates that the flashed targets had to be misaligned in a direction opposite the transformational apparent motion by $21 \mathrm{~min}$ of arc to appear aligned, $t(30)=4.95, p<.05$ (positive values along the abscissa indicate target misalignments opposite the direction of the transformational motion). The bias in the perceived position of the flashed targets depending on the direction of the transformational apparent motion was significant for each subject; the least significant overall effect was for subject E.V., $t(6)=2.24, p<.05$. The width of the flashed target was $\sim 23 \mathrm{~min}$ of arc, so the effect size is comparable to the size of the target.

Figures $2 \mathrm{~B}$ and $2 \mathrm{C}$ show the perceived misalignment between the target flashes for all seven SOAs. There was a significant effect across SOAs for each subject-for D.B., $t(6)=6.68, p<.05$-as well as the group data, $t(3)=4.95, p<.05$. The broad temporal tuning of the effect is further characterized in Experiment 1B.

Figure 2 suggests that transformational apparent motion influences the perceived positions of static targets. How do we know that it is the percept of motion itself that matters-the transformational apparent motion-and not some other feature of the stimulus? For example, spatial allocation or shifts of attention can influence the apparent locations of objects (Pratt \& Turk-Browne, 2003; Suzuki, 2001; Suzuki \& Cavanagh, 1997). A target presented briefly nearby another target can appear displaced in a direction away from the attended location (Suzuki \& Cavanagh, 1997). Suzuki and Cavanagh (1997) argued that the representation of the area around an attended object has a higher resolution. This might result in a kind of distortion that would occur at the attended location and could be akin to cortical magnification (in which a disproportionate area of cortex is devoted to processing the foveal image).

To test the attentional repulsion account, in an additional experimental condition we presented the same $\mathrm{H}$ stimuli (Figure 1) but without a midline; without the midline, there is no motion percept. This is equivalent to presenting the flashed targets well before the second frame (Figure 1C). The open circles in Figure 2B and $2 \mathrm{C}$ show that there was no perceived misalignment in this condition. The most significant effect was -6 min of arc (Figure 2B), which was below threshold and nonsignificant, $t(30)=-0.59, p>.05$. The just noticeable difference (half the distance between the 25 th and 75 th percent response proportions on the psychometric func- tion) was $12 \mathrm{~min}$ of arc for this subject. The results for all 4 subjects were not significant (solid square symbol in Figure 2C), $t(30)=-0.48, p>.05$. The presence of the midline-the second frame of the transformational apparent motion sequence-is therefore necessary to produce the position displacement observed in Experiment 1A. Attentional repulsion alone cannot be responsible.

Experiment 1B: Time Course of Transformational Apparent Motion's Influence on the Positions of Remote Objects

The first experiment established that transformational apparent motion - an illusion of motion without any real motion-caused brief targets to appear shifted in position; the effect was present across the entire range of tested SOAs, from $250 \mathrm{~ms}$ before the illusory moving line to $50 \mathrm{~ms}$ after it. Additional conditions revealed that the effect does not occur because of the static portions of the display (the static stimulus frames do not cause a shift in perceived position). However, it is not yet clear what the time course of the effect is.

The targets in the first experiment were superimposed on the transformational apparent motion-on the midline of the $\mathrm{H}$ stimulus. Previous studies have suggested that low-level motion (luminance defined, detected by passive mechanisms; Whitney, 2002) and high-level motion (detected by top-down mechanisms such as attention; Shim \& Cavanagh, 2004; K. Watanabe et al., 2002 , 2003) influences the perceived positions of targets even when they are separated from the moving stimulus. Because of this similarity among a number of different motion-induced position displacement illusions, it is possible that a common mechanism could be responsible. If so, then transformational apparent motion should also influence the apparent locations of objects at some distance.

The following experiment had two purposes. First, to test whether transformational apparent motion influences position judgments over a distance, we separated the targets from the midline of the $\mathrm{H}$ stimulus so that they were not colocalized with the illusory motion. Second, we tested a broader range of SOAs between the target and the transformational apparent motion to reveal the time course of illusory motion's influence on localization.

\section{Method}

The methods in this experiment were identical to those in the first except the targets were each vertically separated from the midline of the $\mathrm{H}$ stimuli (Figure $3 \mathrm{~A}$, inset) by $3.1^{\circ}$. Also, there were 10 possible SOAs at which targets could be presented (from $1,000 \mathrm{~ms}$ before to $500 \mathrm{~ms}$ after the second frame). All other aspects of the experiment were identical to those in the first experiment.

\section{Results}

Figure 3 shows that there was a perceived shift in the positions of the targets, even though they were separated from the illusory motion of the line. The magnitude of the effect for both D.B. and D.W. exceeded 24 min of arc and was significant for both subjects: for D.B., $t(30)=3.3, p<.05$; for D.W., $t(30)=4.1, p<.05$. Because the perceived misalignment was comparable to that in the first experiment, any scaling with eccentricity (Durant \& Johnston, 
A

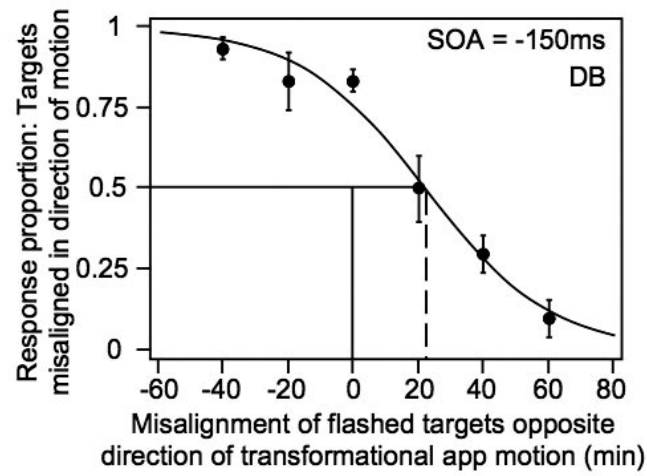

B

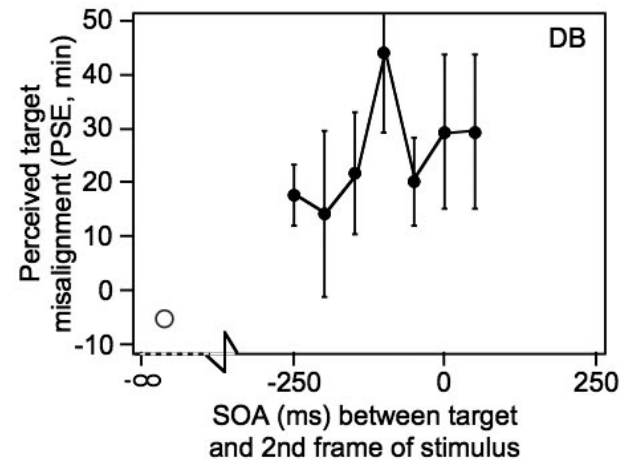

C

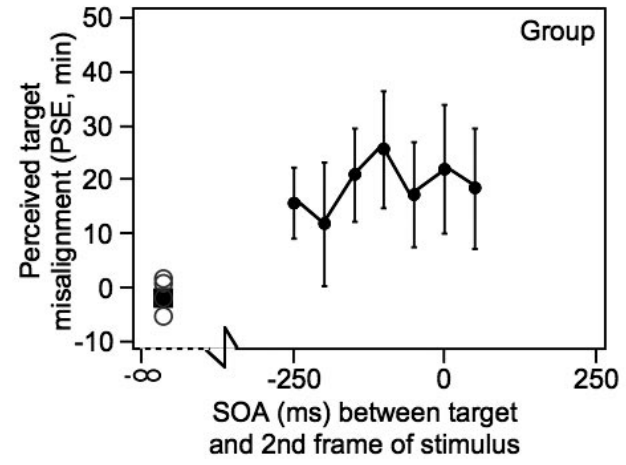

Figure 2. Results of Experiment 1A. A: Representative psychometric function showing the magnitude of the illusory misalignment between the flashed targets when presented $100 \mathrm{~ms}$ before the midline (the midline of the $\mathrm{H}$ stimulus that displays transformational apparent motion). The abscissa shows the misalignment between the two flashed targets; positive values indicate that the targets were misaligned in a direction opposite that of the illusory transformational apparent motion, and negative values indicate that the targets were misaligned in the direction of the motion. The ordinate shows the proportion of the subject's responses that were in the direction of the illusory transformational apparent motion. For example, when the targets were perfectly aligned ( 0 on the abscissa), the subject responded about $85 \%$ of the time that the targets were misaligned in a direction consistent with that of the illusory motion (as shown in Figure 1D). The point of subjective equality (PSE) is indicated by the misalignment (along the abscissa) at which subjects' responses were at 50\% along the ordinate. This is the physical misalignment between the targets that produced an apparent alignment. B: The perceived misalignment (PSE) at various stimulus onset asynchronies (SOAs) between the presentation of the targets and the midline, for subject D.B. An SOA of 0 indicates that the targets were presented simultaneous with the midline; positive SOAs indicate that the flashed targets were presented after the midline (and negative SOAs, before the midline). Each data point represents one PSE,
2004; Whitney \& Cavanagh, 2003; Wright \& Johnston, 1985) is countered by a decrease in the effect with increasing distance from the perceived motion (Durant \& Johnston, 2004). An omnibus analysis of variance (ANOVA) revealed a significant effect for the group, $F(9,39)=2.45, p<.05$.

To characterize the temporal tuning of transformational apparent motion's influence on perceived position, we fit a Gaussian curve to the data in Figure 3 for each subject. Consistent with Experiment 1A, the perceived misalignment is strongest when the flashes precede the second frame by $\sim 80 \mathrm{~ms}$ (cf. Figure 2). The best fit Gaussian, shown for 2 subjects, reveals a broad temporal tuning of the illusion (480-ms full width at half maximum [FWHM] for subject D.W.; 280-ms FWHM for subject D.B.). Figure 3B shows a consistently broad temporal tuning for the group (500-ms FWHM). This indicates that $50 \%$ of the effect is still present $\pm 250 \mathrm{~ms}$ from the peak misalignment. In fact, it is clear that there is a significant perceived misalignment between the targets even when they are presented $\sim 200 \mathrm{~ms}$ before the second frame of the transformational apparent motion sequence; there was a significant effect in that condition for both D.B., $t(30)=3.6, p<.05$, and D.W., $t(30)=$ $3.2, p<.05$, and for the group, $t(3)=5.4, p<.05$. Individual contrasts ( $t$ tests) of the group data revealed that a significant misalignment was perceived when the targets were presented anywhere from $500 \mathrm{~ms}$ before to $50 \mathrm{~ms}$ after the second frame of the transformational apparent motion display; the least significant of these contrasts was for the -100 -ms condition, $t(3)=$ $2.4, p<.05$. An interesting point here is that the perceived position of the flashed object in this experiment requires several hundred milliseconds to pass before the visual system assigns its position. This is comparable to previous experiments showing that physical motion (as opposed to strict illusory motion) influences perceived position on a similarly long time scale (Whitney \& Cavanagh, 2000).

Our use of a stimulus that induces a percept of motion without the presence of real motion lends strong support to the idea that perceived motion in one location can influence the perceived position of an object in another location, extending previous examples of top-down motion-induced position shifts (Shim \& Cavanagh, 2004; K. Watanabe et al., 2002, 2003). The question remains whether all motion-induced position displacement illusions are due to a single top-down mechanism. This possibility is directly tested in Experiment 2.

derived from a psychometric function like that in (A). At SOAs around 0 , the flashed targets appeared shifted in the direction of the illusory motion. Targets presented when only the first frame was visible (open symbol) did not appear misaligned. $\mathrm{C}$ : The perceived misalignment as a function of SOA averaged across all subjects. In addition to the within-subject effects, there is a significant main effect of the illusory motion on the perceived positions of the targets for the group, $t(3)=4.95, p<.05$. Targets presented when only the first frame was visible did not appear misaligned (open symbols and solid square). Error bars show 95\% confidence intervals. app $=$ apparent. 
A

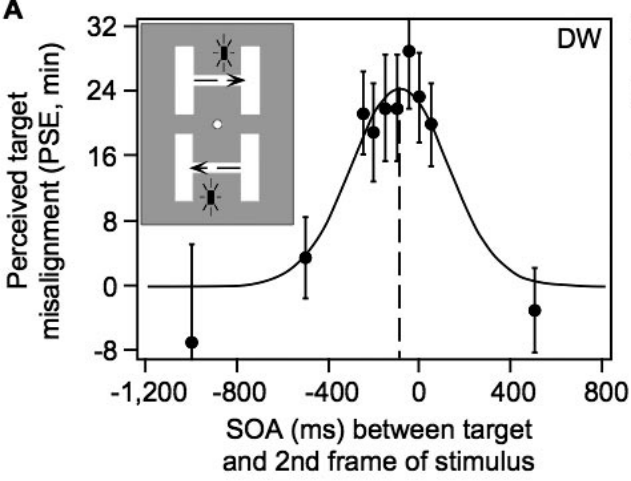

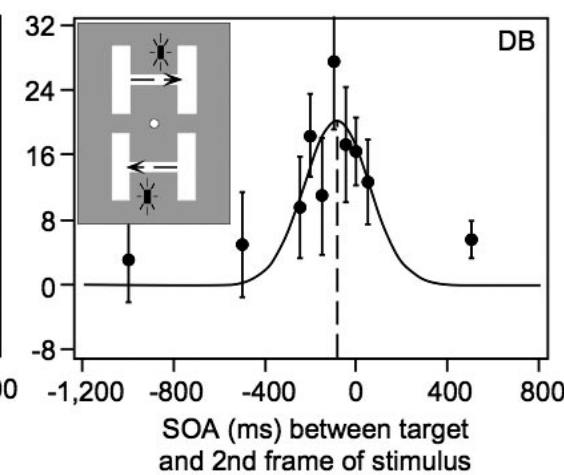

and 2 nd frame of stimulus

B

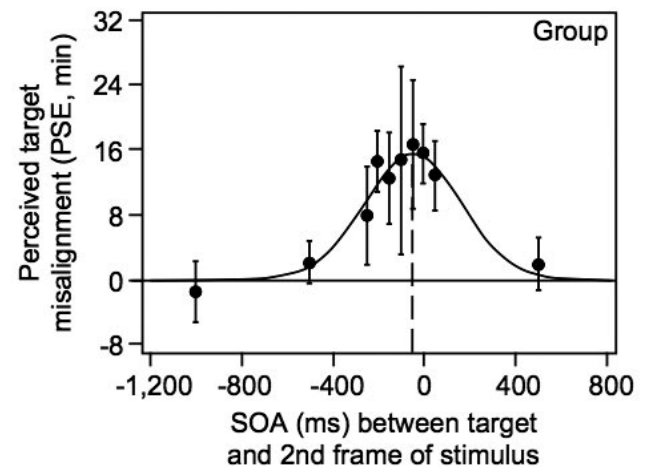

Figure 3. Results of Experiment 1B. A: The stimulus and percept in Experiment 1B (inset) was similar to that in Experiment 1A, shown in Figure 1, except that the targets were vertically separated from the illusory motion in the display. The perceived misalignment was measured at various stimulus onset asynchronies (SOAs) before and after the second frame of the transformational apparent motion sequence. Each data point represents the point of subjective equality (PSE) of a psychometric function. Ten PSEs were measured for each subject to assess the temporal tuning of transformational apparent motion's influence on perceived position. When the targets were presented at an SOA of $-500 \mathrm{~ms}$ (or prior to that), there was no perceived misalignment between the targets. Likewise, targets presented more than $400 \mathrm{~ms}$ after the second frame of the sequence were perceived veridically. Perceived position therefore depends on information within a restricted temporal window. To measure the temporal tuning of the effect, a Gaussian was fit to the data for each subject: $f(x)=[a \cdot \exp (-(x+$ $\left.b)^{2} / c^{2}\right)$ ], where $a$ is the peak misalignment perceived between the targets, $b$ is the temporal shift in the peak effect relative to $0 \mathrm{SOA}$, and $c$ is the width of the Gaussian at $78 \%$ maximum amplitude. The best fit parameters for subject D.W. were $a=22.8 \mathrm{~min}$ of arc, $b=-84.8 \mathrm{~ms}$, and $c=300 \mathrm{~ms}$. The $r^{2}$ of the fit was .92 . The best fit parameters for subject D.B. were $a=18.9 \mathrm{~min}$ of arc, $b=-87.9 \mathrm{~ms}$, and $c=201 \mathrm{~ms}$. The $r^{2}$ of the fit was .62. B: The perceived misalignment between the targets as a function of SOA for all subjects averaged. The best fit parameters were $a=15.5 \mathrm{~min}$ of arc, $b=-48 \mathrm{~ms}$, and $c=300 \mathrm{~ms}$, indicating that the peak misalignment occurred about $50 \mathrm{~ms}$ before the presentation of the second frame of the transformational apparent motion sequence. The $r^{2}$ of the fit was .95. The effect was broadly tuned in time, showing that half of the effect was present more than $200 \mathrm{~ms}$ before the presentation of the second frame of the sequence. Evidently, the visual system integrates information over a long temporal interval before assigning positions to objects. Error bars show $95 \%$ confidence intervals.

\section{Experiment 1C: Is a Frame of Reference Illusion or the Percept of Motion Necessary?}

The midlines of the $\mathrm{H}$ stimuli presented in Frame 2 (see Figure 1) were physically misaligned (i.e., the new information visible in Frame 2 consisted of two midlines that were offset relative to each other): This offset could induce a tilt illusion or frame of reference effect (Duncker, 1929/1938; Rock, 1990; Roelofs, 1935; Witkin \& Asch, 1948), which could cause the two flashed targets to appear misaligned, independent of any motion percept. To test whether a frame of reference effect is necessary or whether the percept of transformational apparent motion is responsible for the data in
Figure 3, we modified the stimulus in Figure 1 slightly to remove the frame of reference information.

\section{Method}

The methods in this experiment were identical to those in Experiment 1B except that the stimulus was modified slightly. Figure 4A shows that one of the vertical stalks of the $\mathrm{H}$-shaped stimuli was wider than the other; in fact, the width perfectly matched the width of the cue. Therefore, the horizontal midline (the dynamic part of the stimulus that displays an illusory motion) was vertically aligned in the top and bottom displays (Figure 4B). All other details of the experiment were identical to those in Experiment 1B. 

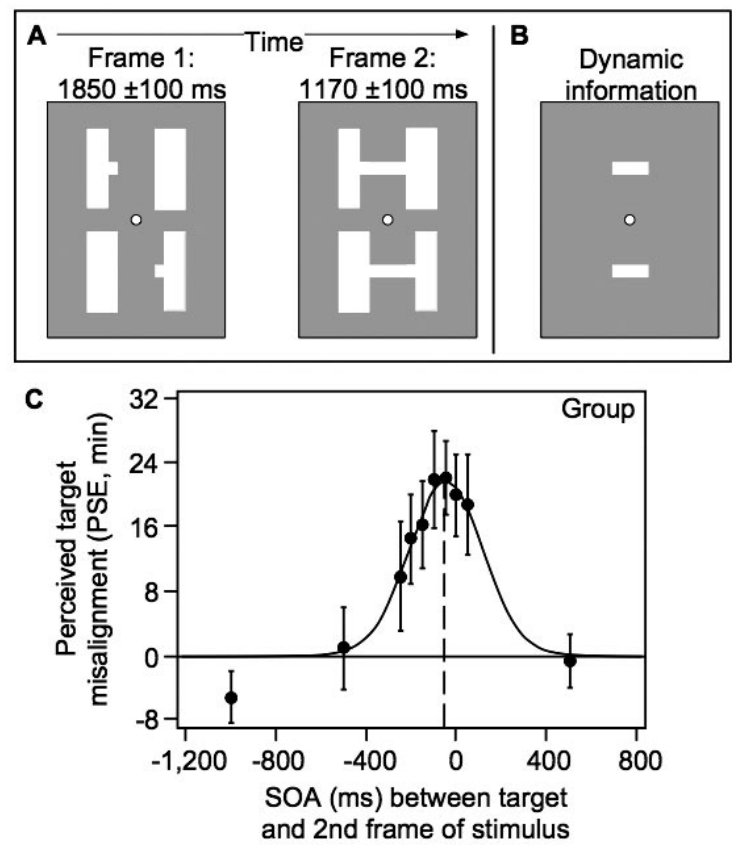

Figure 4. Stimulus and results for Experiment 1C. A: The transformational apparent motion stimulus from Figure 1 was slightly modified. The midline that displays transformational apparent motion was vertically aligned by making one vertical stalk of each $\mathrm{H}$ stimulus wider. B: The resulting transformational apparent motion occurs across two vertically aligned segments, eliminating any tilted frame of reference that may have been present in the dynamic part of the original display in Figure 1. C: Averaged data across 4 subjects showing a perceived misalignment between the target flashes comparable to Figure 3B. The best fit Gaussian (from Figure 3) had a peak shift of $\sim 48 \mathrm{~ms}$, comparable to the first experiment. The results demonstrate that a frame of reference or tilt illusion is not responsible for the results. Likewise the static frames themselves (Frame 1 or Frame 2) are not responsible for the results, because no effect was found well before or well after the transformational apparent motion (i.e., at $-1,000-\mathrm{ms}$ and at $500-\mathrm{ms} \mathrm{SOA}$, there is no perceived misalignment). Error bars show 95\% confidence intervals.

\section{Results}

Figure $4 \mathrm{C}$ shows that there was a significant misalignment perceived between the two flashed targets, even though the dynamic part of the display was perfectly aligned, $F(9,39)=4.22$, $p<.05$. The temporal tuning of the effect was consistent with that found in Experiment 1B, as revealed by the best fit Gaussian. Because the horizontal midlines between the $\mathrm{H}$ stimuli that display transformational apparent motion are vertically aligned, a frame of reference effect cannot be present. One might argue that the first and second frames themselves (as a whole) are now spatially asymmetric and that these static frames (either Frame 1 or Frame 2) could contribute to the results in Figure 4 . We can rule this possibility out, however, because well before the second frame (e.g., $-1,000-\mathrm{ms} \mathrm{SOA}$ ), there is no perceived misalignment between the flashed targets. Likewise, well after the second frame (500-ms SOA), there is also no misalignment perceived. Therefore, a frame of reference effect like the Duncker or Roelofs illusion (Duncker, 1929/1938; Rock, 1990; Roelofs, 1935; Witkin
\& Asch, 1948) cannot be responsible for the results. Rather, the percept of illusory transformational apparent motion is necessary.

\section{Discussion: Experiments 1A-1C}

The first experiment demonstrated that the perception or awareness of motion direction is sufficient to shift the apparent locations of objects, regardless of whether real motion is present in the scene. Consistent with previous studies (Shim \& Cavanagh, 2004; K. Watanabe et al., 2002, 2003), high-level or top-down motion detection mechanisms must contribute to perceived position. Alternative stimulus-driven mechanisms, such as passive first-order motion detectors, can be ruled out because the stimulus in Figure 1 contains no motion (not even ambiguous/bistable motion or a change in the position of a target over time). When only parts of the display were presented (at the extreme SOAs), there was no effect on the perceived position of the static target. Therefore, effects such as attentional repulsion (Pratt \& Turk-Browne, 2003; Suzuki \& Cavanagh, 1997) and frames of reference (Duncker, 1929/1938; Rock, 1990; Roelofs, 1935; Witkin \& Asch, 1948) can also be ruled out because the illusory position shift occurred only when transformational apparent motion was perceived. The shift is not an example of the onset repulsion (Thornton \& Hubbard, 2002) or flash-lag effects (Krekelberg \& Lappe, 2001; Nijhawan, 2002), because the shift is in the direction of motion and occurs for a static object.

The advantage of using transformational apparent motion over other types of stimuli used in the past is that most forms of illusory motion require a change in the position of an object, or feature, over time. For example, bistable apparent motion-even longrange apparent motion-constitutes a change in the position of an object over time. Biasing attention toward one direction of motion influences the apparent positions of static objects (Shim \& Cavanagh, 2004), but this may be due to a modulating role of attention rather than a top-down mechanism that mediates position assignment. Anorthoscopic motion (motion of an object only visible through a pixel-wide slit; K. Watanabe et al., 2002) reduces directional motion signals because of the aperture problem that is created in those displays; integration over space, however, solves the aperture problem. The true direction of object motion could thus be solved in a bottom-up fashion. Transformational apparent motion also provides stronger evidence for a top-down contribution to perceived position than the line-motion illusion because the latter illusion has two temporally restricted events (a flash followed by a briefly presented line; Hikosaka, Miyauchi, \& Shimojo, 1996), which could potentially be detected by passive motion detectors (Zanker, 1997). The perceived direction in transformational apparent motion displays, on the other hand, depends on a surface or form interpretation (Tse \& Logothetis, 2002).

The mechanism underlying transformational apparent motion certainly requires attention or top-down knowledge (Tse \& Logothetis, 2002), though the precise implementation is still unknown. It could be related to inferred motion (Assad \& Maunsell, 1995), reduced latency at the attended (cued) location (Baldo \& Klein, 1995; Hikosaka, Miyauchi, \& Shimojo, 1993; Stelmach \& Herdman, 1991), surface filling in and boundary completion (Baloch \& Grossberg, 1997; Downing \& Treisman, 1997), form-specific motion processing (Tse \& Logothetis, 2002), or attentive tracking (Cavanagh, 1992), and it may involve multiple independent pro- 
cesses (von Grünau, Dube, \& Kwas, 1996). Regardless of the specific mechanism that gives rise to transformational apparent motion, a top-down component is necessary to explain the illusory motion percept (Tse \& Logothetis, 2002). Therefore, we can safely conclude that because nothing in the stimulus actually moved or changed position, the perception or awareness of motion direction is sufficient to cause objects to appear shifted.

\section{Experiment 2A: Is Attention to or Awareness of Motion Direction Necessary?}

In the following experiment we tested whether the position of a target can be shifted by motion even when the direction of motion cannot be identified. We used the well-known phenomenon of crowding (Bouma, 1970, 1973; Levi, Klein, \& Aitsebaomo, 1985; Stuart \& Burian, 1962). In crowding displays, the number and spacing of an array of objects is such that observers are unable to report or scrutinize the specific features of the objects near the center of the array (Felisberti, Solomon, \& Morgan, 2005; He, Cavanagh, \& Intriligator, 1996; Pelli, Palomares, \& Majaj, 2004). Although observers clearly see that something is there, they are unable to identify the crowded objects, how many there are, or any other specific features of the objects (He, Cavanagh, \& Intriligator,
1997; Kim \& Blake, 2005). Despite the fact that observers are unable to scrutinize or discriminate the features of a crowded object, several studies have shown that low-level (bottom-up) adaptation occurs and is specific to these unidentifiable features (Aghdaee, 2005; Aghdaee \& Zandvakili, 2005; He et al., 1996; Rajimehr, Montaser-Kouhsari, \& Afraz, 2003; Rajimehr, VaziriPashkam, Afraz, \& Esteky, 2004). For example, a crowded array of oriented gratings can prevent subjects from identifying the orientation of the central-most pattern; nevertheless, there is still orientation-specific adaptation and aftereffects (He et al., 1996, 1997). Likewise, adapting to moving patterns embedded in crowded arrays can prevent awareness of motion direction while still producing direction-specific motion aftereffects (Aghdaee, 2005; Aghdaee \& Zandvakili, 2005; Whitney, 2005). We adopted this technique by presenting an array of Gabor stimuli that contained patterns drifting in random directions (Figure 5). We then tested whether the crowded Gabors - the ones whose direction of motion was unidentifiableinfluenced subsequent judgments of perceived position.

\section{Method}

Four subjects participated in the experiment (the same from the first experiment, 3 of whom were naive). Each had normal or corrected-to-

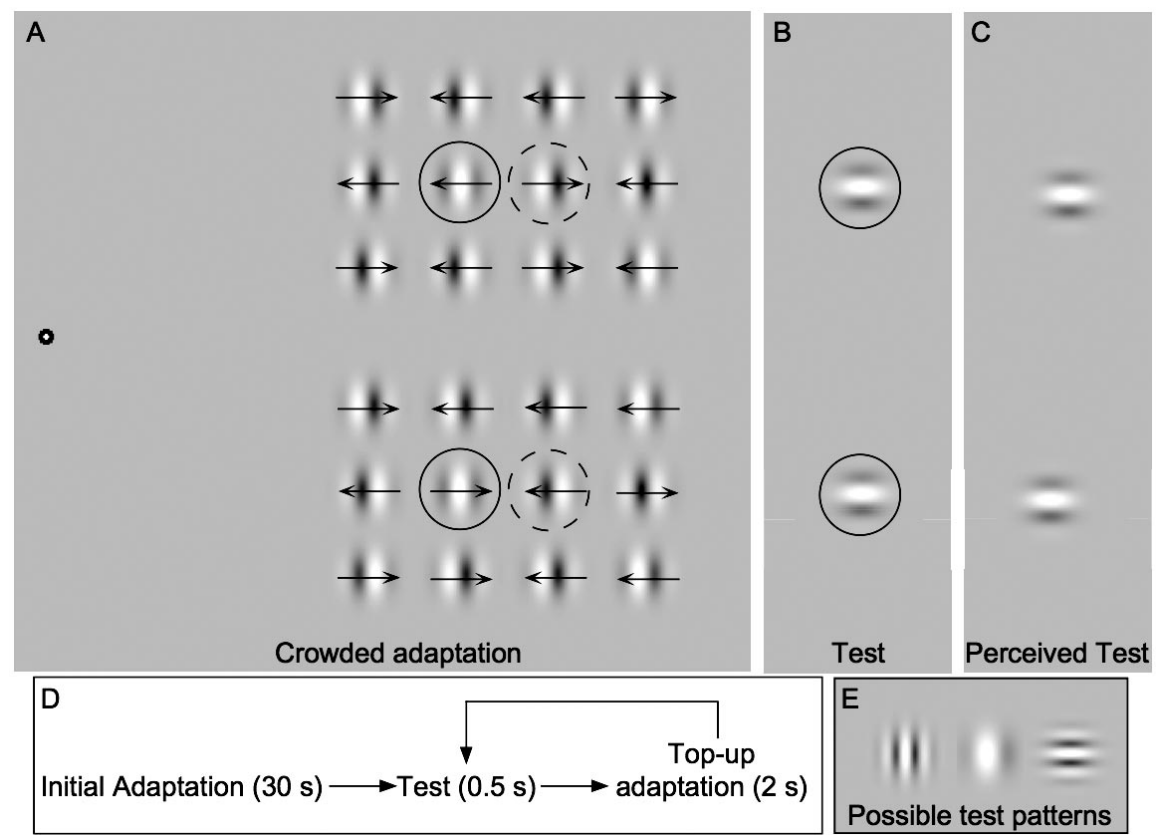

Figure 5. Stimuli used in the second experiment. A: An array of Gabor patches was presented for an adaptation period while subjects fixated on the bull's-eye. Two pairs of vertically aligned Gabors in the central region of the array had opposing directions of motion. These adaptation regions are circled by dashed and solid lines, respectively (these circles are for illustration and were not presented in the actual stimulus). The directions of motion in the adaptation regions (circled) were fixed throughout an experimental session. All other Gabors served as crowding stimuli and had randomly determined directions of motion (leftward or rightward) on each trial. B: During the test period, two static Gabors were presented in one of the two vertically aligned, adapted regions (e.g., the test Gabors are located within the circled regions that were previously adapted to motion, in [A]). C: After adapting to the stimulus in (A), the test Gabors in (B) appear to be misaligned in a direction opposite that of the prior motion adaptation. D: Each session began with an initial adaptation period, followed by a repeat test and top-up adaptation periods. E: Examples of several different kinds of test Gabors that could be presented during the test period. 
normal vision. Subjects were seated in a darkened experimental booth with a chin rest $28 \mathrm{~cm}$ from a Sony G520 Multiscan CRT monitor, which had a refresh rate of $100 \mathrm{~Hz}$.

Stimuli were similar to that shown in Figure 5 and consisted of an array of drifting Gabor patterns (moving sine wave carriers with static Gaussian envelopes) on a gray $\left(52.5 \mathrm{~cd} / \mathrm{m}^{2}\right)$ background. The array contained 24 approximately equally spaced Gabors (horizontal center-to-center separation between the Gabors was $4.6^{\circ}$; vertical center-to-center separation was $\left.4.2^{\circ}\right)$. Each Gabor had a contrast of $99 \%$, had 0.52 cycles/deg, drifted at 6 cycles/s, and had a Gaussian envelope with a standard deviation of $0.74^{\circ}$.

In the central region of the array were two pairs of vertically aligned Gabors whose direction of motion was always opposite (the vertical pairs are circled with dashed and solid lines, respectively, in Figure 5A; these circles are for illustration and were not presented in the actual stimulus). The direction of motion in these adapted regions was always the same across trials, though subjects were not able to identify the direction of motion in these adapted regions (direct tests of this are provided in Experiment 2C; see also Experiment 2E). Each of the other surrounding Gabors (20 of them) had either leftward or rightward motion on each trial, determined randomly for each one on each trial, which amounted to a net global velocity of zero across trials. A fixation bull's-eye was provided $23^{\circ}$ to the left of the leftmost test Gabor in the array. Subjects were instructed to fixate the bull's-eye at all times.

On each trial, the array of Gabors was presented for $2 \mathrm{~s}$ (an adaptation period). A pair of stationary test Gabors was then presented for $500 \mathrm{~ms}$ in one of the two vertically aligned adapted regions (Figure 5B; vertical separation between the test Gabors was $15.1^{\circ}$ ). The orientation of the test Gabors was either the same as or orthogonal to (rotated $90^{\circ}$ ) the Gabors in the array. The test Gabors had one of three spatial frequencies, determined randomly on each trial $(0.35,0.52$, or 1 cycle/deg). Rotating the carrier eliminates the perceived motion aftereffect (McGraw et al., 2002). The phase of the test Gabor's carrier was randomized on each trial. The test Gabors were vertically aligned (as shown in Figure 5B) or could be physically misaligned by one of six possible values (at increments of $0.46^{\circ}$, for a total of seven possible relative alignments). The adapted region in which the test Gabors were presented and the misalignment between them were randomized on each trial. In a two-alternative forced-choice (2AFC) method of binary choice (yes-no) task, subjects were asked to judge the relative alignment of the test Gabors (whether the top Gabor was to the left or to the right of the bottom one). Immediately after subjects responded, another trial was presented. Because the two vertically aligned pairs of adapting Gabors contained motion in opposite directions (Figure 5A), the perceived misalignment between the test Gabors was in opposite directions depending on where the test Gabors were presented (Figure 5B-C). The results for these two directions of adaptation were comparable, so the data were flipped and merged across adaptation directions.

In each experimental session, there were two possible test Gabor orientations (orientation was either the same as or orthogonal to that of the adapting stimuli), three possible spatial frequencies, two possible adaptation positions, seven possible flash alignments at each adaptation position (i.e., aligned, or misaligned by one of six values), and 10 repeated trials for each of these seven flash alignments, for a total of 840 trials per session (broken up into two equal length subsessions because of the duration). Data for the two adaptation positions were collapsed, and for each test orientation and spatial frequency, a psychometric function was fit to the data from the logistic function described in Experiment 1A. The PSE reflects the sum of the two Gabors' position shifts (i.e., the physical distance between the two test Gabors). For example, when the test Gabors were physically aligned, they each appeared shifted in a direction opposite that of the prior motion adaptation (Figure 5C); in order to offset this apparent misalignment, the test Gabors had to be physically misaligned. In separate sessions, the direction of the four adaptation Gabors was reversed (Figure 5 shows only one direction of adaptation; to avoid any adaptation asymmetry, the direction of the adaptation Gabors was reversed in an additional session).
Each subject participated in three separate sessions $(840 \times 2$ directions of adaptation $\times 3$ sessions $=5,040$ trials), yielding three psychometric functions for each type of test Gabor. The PSE for each condition was based on the average of the three psychometric functions.

In a separate test, using the same subjects and methods above, the stationary test Gabors were presented for $100 \mathrm{~ms}$ (rather than $500 \mathrm{~ms}$ ). All other details of this second manipulation were identical to those above.

\section{Results}

Figure $6 \mathrm{~A}$ shows that after adaptation to crowded motion, there was a significant shift in the perceived positions of stationary test Gabor stimuli presented within the adapted regions: for subject D.B., $t(30)=4.6, p<.05$; for subject D.W., $t(30)=3.2, p<.05$. The psychometric functions for two representative subjects show that the PSE shifted by $\sim 0.6^{\circ}$ each after motion adaptation (Figure $6 \mathrm{~A})$. Figure $6 \mathrm{~B}$ shows that the illusory position shift was comparable across different spatial frequencies, suggesting that the motion adaptation is not pattern specific, in contrast to many types of firstorder motion aftereffects (MAEs; Mather et al., 1998). A betweensubjects ANOVA revealed a significant main effect of motion adaptation on the perceived positions of the test stimuli, $F(1,3)=21, p<$ .05 . Post hoc contrasts indicated that there was a significant influence of motion adaptation on perceived position for each of the subjects; least significant effect was for E.V.: $t(6)=5.96, p<.05$.

When the orientation of the test Gabors was identical to that of the adaptation Gabors, there was a strong MAE that accompanied the apparent position shift. This MAE was produced even though the drifting Gabors were crowded during adaptation. To mitigate the possibility that the perceived shift in the position of the test Gabor was due to the perception of the MAE, we reduced the duration of the test Gabors to $100 \mathrm{~ms}$ in a separate test, as this reduces the perceived MAE (Nishida \& Johnston, 1999; Whitney \& Cavanagh, 2003). The perceived shift in the position of the test Gabors was still significant $\left(0.25^{\circ}\right)$ even with this short test duration; for representative subject D.W., $t(60)=2.6, p<.05$.

To further ensure that the perception of a passive MAE is not responsible for the illusory position shift, we presented one condition in which the test Gabors had an orientation orthogonal to that of the adaptation Gabors. This eliminates the MAE (McGraw et al., 2002) or at least dramatically reduces it, but leaves intact the apparent shift in the position of the test Gabor (Figure 6B, solid symbols). In these orthogonal test Gabors, there was still a significant shift in their perceived positions, $t(3)=4.8, p<.01$. There was not a significant effect of test Gabor spatial frequency on the perceived misalignment, $F(2,6)=2.6, p=.17$.

It is noteworthy that the orthogonally oriented test Gabors showed a reduced effect $\left(\sim 0.5^{\circ}\right.$ misalignment for the orthogonal test Gabors as compared with $\sim 0.7^{\circ}$ for the parallel test Gabors). This main effect difference between parallel and orthogonally oriented test Gabors did not reach significance, $t(3)=2.75, p=.07$, but the effect size $\left(\mathrm{eta}^{2}=\right.$ 0.7) suggests that the orthogonal carrier did reduce the magnitude of the perceived misalignment. It is possible, if not likely, that this is due to the reduced magnitude of the perceived MAE. This raises the possibility that the misalignment perceived between the test Gabors is produced by the percept of motion (the MAE). In the following experiment, we determine whether perceived motion during the test period is responsible for the perceived shift in the position of the Gabors by nulling the perceived MAE and then measuring whether an illusory shift still remains. 


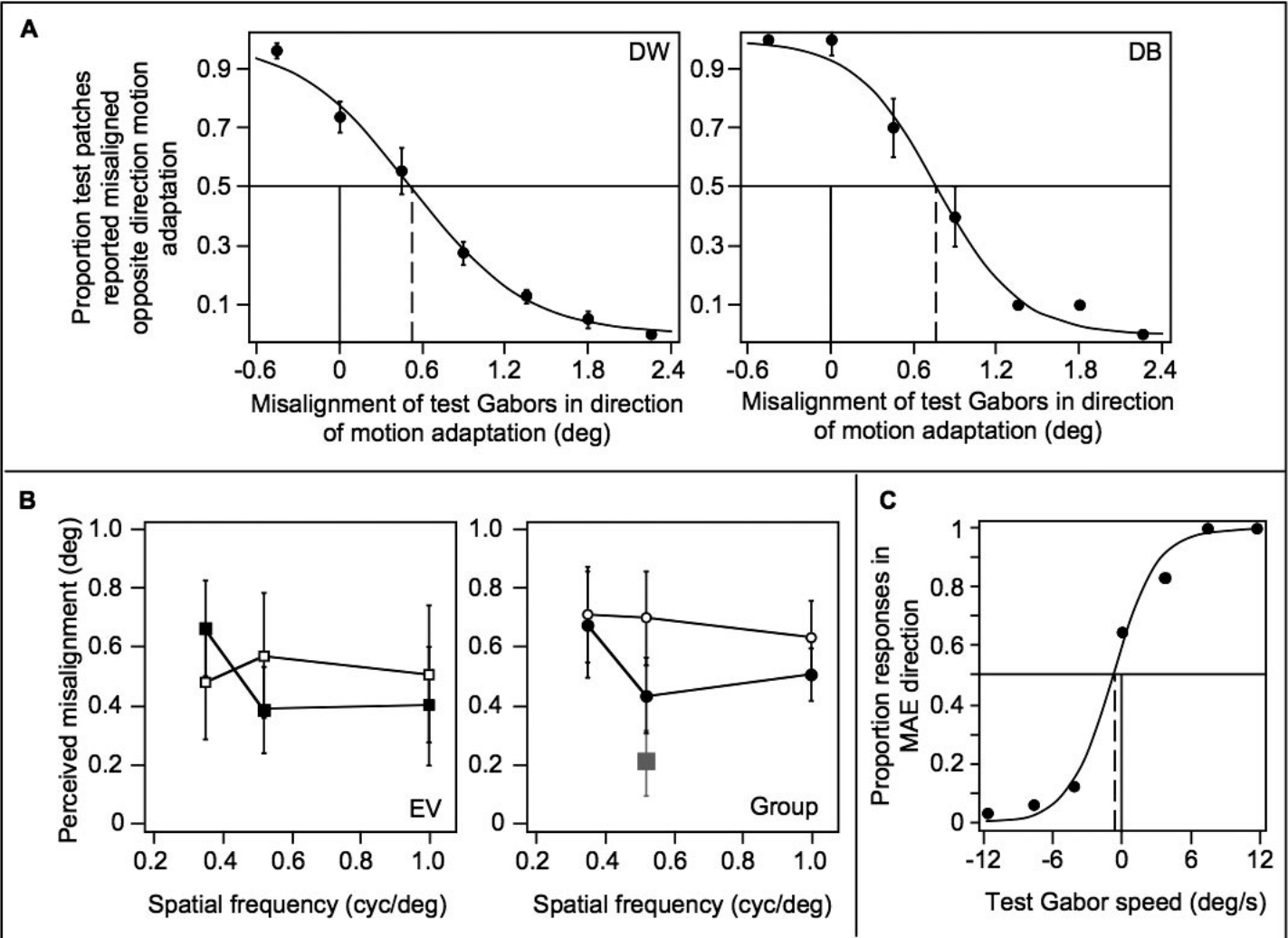

Figure 6. Results of Experiment 2A. A: Representative psychometric functions for 2 subjects showing a significant shift in the perceived position of the test Gabors as a function of the direction of motion adaptation. The abscissa shows the physical misalignment between the test Gabors: Positive values indicate that the Gabors were misaligned in the same direction as the prior motion adaptation, and negative values indicate that the Gabors were misaligned in a direction opposite that of the motion adaptation. The ordinate shows the proportion of trials in which the subject perceived the test Gabors to be misaligned opposite the direction of the prior motion adaptation. The point of subjective equality defines the physical misalignment between the Gabors that appeared to be aligned. Because of the motion adaptation, the Gabors had to be presented $\sim 0.6^{\circ}$ in the same direction as the prior motion adaptation to appear aligned. When physically aligned, the Gabors appeared shifted opposite the direction of the motion adaptation. B: Results for one representative subject (left panel) as well as for all 4 subjects averaged (right panel) as a function of the spatial frequency (abscissa) and orientation of the test Gabors. The orientation of the test Gabors was either the same as (open symbols) or orthogonal to (solid symbols) the orientation of the adaptation Gabors. There was a significant overall position shift for all subjects; least significant effect was for subject E.V., $t(5)=5.96, p=.002$. There was a larger effect when the adaptation and test Gabors contained the same orientations; for the group results in the right panel, $t(3)=2.75, p=.07$. There is little variation in the illusory position shift as a function of spatial frequency. Error bars indicate within-subject SEM in the left panel and between-subjects SEM in the right panel. C: Results of Experiment 2B, showing the perceived motion in a single orthogonally oriented test Gabor. The perceived motion for this representative subject was $0.4 \%$, which although not significant, $t(2)=1.3, p>.05$, could have determined the misalignment in (B) above. The solid gray square in the right-hand panel of (B) shows the perceived misalignment when the test Gabor is drifted at a speed that nulls the perceived motion aftereffect (MAE). There is still a perceived misalignment even with the MAE nulled, $t(5)=3.32, p<.05$. This demonstrates that neither the percept of motion nor a subthreshold MAE is responsible for the illusory mislocalization.

\section{Experiment 2B: Position Shifts Without Perceived Motion}

There is a possibility that the perceived misalignment between the test Gabors in Figure 6 could have been caused by a perceived MAE during the test period. If this were the case, a high-level motion mechanism could explain the result. If, however, a perceived misalignment between the test Gabors occurs without any percept of motion (real or illusory), then we can be confident that bottom-up motion detectors are responsible and that the awareness of motion direction must not play a role.

\section{Method}

There were two parts to this experiment. In the first, Experiment 2A was repeated, except that the perceived motion of the test Gabors was measured rather than their perceived misalignment. Only one condition was tested 
( 0.52 cycles/deg Gabors; the test Gabor's carrier was orthogonal). Following adaptation as in Experiment 2A, a single orthogonally oriented test Gabor was physically drifted (the whole pattern moved, using subpixel motion; Georgeson, Freeman, \& Scott-Samuel, 1996; Mather \& Morgan, 1986; Morgan, Mather, Moulden, \& Watt, 1984) at one of six different speeds (ranging from $-12^{\circ}$ to $12^{\circ}$ in equal increments). Using the method of constant stimuli (leftward-rightward judgment), we measured the speed and direction of test Gabor motion that nulled the perceived MAE (the PSE on the psychometric function). The adaptation Gabors were identical to those described above, except that the direction of motion in the crowded Gabors was randomized (rather than being fixed on every trial), as this reduces the buildup of motion adaptation (note that the goal here was to minimize the MAE). The test Gabor (100-ms duration) was slightly larger than the adaptation Gabors (the Gaussian envelope had a $1^{\circ}$ standard deviation), as this also reduces perceived MAE.

The PSE in the design above reveals the MAE perceived on the test Gabor. In the second part of the experiment, we used this value to null the perceived MAE and then measure whether a position shift remained. In this second experimental session, the perceived misalignment between two test Gabors was measured. The stimulus was identical to that described in the first part above except that two test Gabors were presented and, rather than measuring the perceived MAE, the perceived misalignment between the test Gabors was measured. The method was identical to that of Experiment $2 \mathrm{~A}$ with the important exception that the two test Gabors were physically drifted at a speed that nulled the perceived MAE (as measured above in the first part)

\section{Results}

Figure $6 \mathrm{C}$ shows that the perceived MAE in the orthogonal test Gabors was negligible. The perceived speed of $\sim 0.4 \%$ is well below the just noticeable difference (measured as half the distance between the 25th and 75th percent response proportions on the psychometric function, which was $\sim 4.5 \%$; the high threshold is due to the brevity of the test Gabor; Cropper \& Derrington, 1996; Festa \& Welch, 1997). Because subthreshold motion could still generate a position shift, the second part of this experiment nulled the perceived MAE, and the perceived misalignment between the Gabors was measured. The solid gray symbol in Figure 6B shows that in this situation, although no illusory MAE is perceived, there was still a small shift in the positions of the test Gabors $\left(\sim 0.22^{\circ}\right)$. Although slight, the effect is significant, $t(5)=3.32, p<.05$, demonstrating that an MAE is not necessary to shift the perceived positions of objects. Rather, adaptation to motion can produce effects on apparent location without requiring a percept of motion. These results further suggest that there is a passive, bottom-up contribution of motion (local motion adaptation) to perceived position that operates independent from the mechanism responsible for perceived motion.

\section{Experiment 2C: Efficacy of Crowding During Motion Adaptation}

The preceding experiment demonstrated that exposure to motion in crowding conditions can influence subsequent position judgments. This crowding technique, however, hinges on the assumption that subjects could not detect the direction of motion in the adaptation Gabors. If subjects were able to discriminate the direction of motion, this could influence the subsequent position aftereffect. In this experiment, we sought to test this premise directly by measuring the degree of crowding.

\section{Method}

Stimuli were identical to those in Experiment 2A. The only difference was that subjects were instructed to judge the direction of motion in one of the adapted regions. Because there are two adapted regions-solid or dashed circled stimuli in Figure 5A-subjects were instructed to judge the direction of motion in one region (e.g., the closer Gabor-the one located two from the left and two from the top) in one session and then judge the other region in a subsequent session. Responses were then averaged across these two sessions. On each trial, the fixation bull's-eye was placed a random distance between $12^{\circ}$ and $30^{\circ}$ from the nearest adapted region within the crowded display (the original distance was $\sim 23^{\circ}$ ). The fixation point was always located on the same horizontal plane as in Experiment $2 \mathrm{~A}$ (i.e., it was only displaced along a horizontal dimension). The direction of Gabor motion in the adapted regions (circled in Figure 5A) was random on each trial, which forced subjects to guess the direction of motion on each trial. The direction of all other Gabors was also randomly determined on each trial (leftward or rightward). Subjects responded with one of two keys indicating that the adapted region contained leftward or rightward motion. There were 20 trials for each fixation position, six fixation positions, and two sessions, for a total of 240 trials for each subject.

\section{Results}

Figure 7 shows the subjects' accuracy when guessing the direction of motion in the adapted regions (averaged across both regions circled in Figure 5A). Because of the crowding and depending on

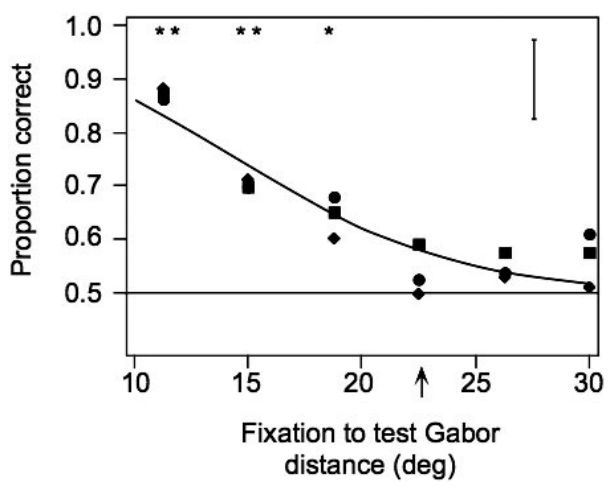

Figure 7. Results of Experiment 2C: Accuracy of discriminating Gabor motion under crowding conditions for 3 experienced subjects. The separation between the fixation point and the test Gabor is plotted along the abscissa. The test Gabor was always located in one of the adaptation regions from Experiment 2A (one of the circled regions in Figure 5). The test Gabor was always embedded in the array of crowding Gabors, and subjects often reported guessing the direction of motion in the test Gabor (i.e., subjects could not even resolve which Gabor was the one to judge because of the crowding). The proportion of responses that were correct is plotted along the ordinate. When the fixation point was close to the array of Gabors, it was easy for subjects to judge the direction of motion within the test Gabor (chi-square test: $* p<.05$; $* * p<.01$ ). When the fixation point was at least $21^{\circ}$ from the nearest test Gabor, subjects were at chance when guessing the direction of motion in the test Gabors (chi-square test: $p>.05)$. In Experiment $2 \mathrm{~A}$, the distance between the fixation point and the test Gabors was $\sim 23^{\circ}$. At this separation (indicated by the arrow), subjects were unable to reliably determine the direction of motion in the test Gabors. The best fit logistic function for the averaged data (solid line) was $f(x)=[1 /(2+\exp (0.21(x-11.25)))+0.5]$, indicating that to achieve $\sim 83 \%$ correct, the fixation point had to be within $11.25^{\circ}$ of the target Gabor. Representative error bar indicates \pm 1 SEM. 
the distance between fixation and the adaptation Gabor, subjects found the task difficult and reported guessing a large proportion of the time. When the fixation point was less than $15^{\circ}$ away from the adaptation Gabors, subjects were at least $70 \%$ accurate; at $15^{\circ}$ separation, the least significant subject was significantly above chance: $\chi^{2}(1,40)=6.4, p<.05$. With separation of more than $20^{\circ}$, however, accuracy rates were around $55 \%$, with each subject responding near chance; subject D.B.'s accuracy (diamond symbols in Figure 7), averaged over all separations above $20^{\circ}, \chi^{2}(1$, $120)=1.6, p=.21$. The separation in Experiment $2 \mathrm{~A}$ was $\sim 23^{\circ}$, suggesting that subjects were unable to determine direction of motion in the adaptation Gabors in that experiment. The crowding stimulus (Figure 5) was therefore an effective manipulation to prevent awareness of motion direction during adaptation.

\section{Experiment 2D: Randomizing the Direction of Motion Across Trials}

There are two more possible ways that subjects could have become aware of motion direction during adaptation. One might argue that although the motion of the adaptation Gabor was indiscernible in Experiment 2A, the Gabor patterns at the edge of the array could be perceived and thus influence the misalignment. This possibility can be ruled out because the Gabors at the edges (and indeed everywhere except the specific adaptation regions) did not contain consistent directional motion, which would prevent adaptation across trials and would not predict the misalignment that subjects perceived between the test Gabors.

Another possibility is that subjects somehow became aware of the direction of motion in the two pairs of adaptation Gabors (circled in Figure 5A). Because the direction of motion adaptation was consistent across trials, if subjects were able to determine the direction of motion just once, they might maintain this awareness throughout the rest of the session. One way that subjects could have become aware of the adaptation direction is by moving their eyes. This scenario is unlikely, however, because we provided a fixation point and our observers were highly trained. Another possibility is that the crowding in the display could have occasionally failed. A crowded stimulus can become visible (crowding can break down) if the stimulus has dramatically different features from the surrounding objects (Chung, Levi, \& Legge, 2001; Kooi, Toet, Tripathy, \& Levi, 1994); for example, a feature that is unique can pop out of a visual search display even when the stimulus would otherwise be crowded out of awareness. In Experiment 2A, the direction of motion was random for each of the crowding Gabors (leftward or rightward), which means that there was a slim probability that many or all of the crowding Gabors could move in the same direction; one of the adaptation Gabors, moving in the opposite direction, might have therefore popped out and become visible. If this (or some other similar perceptual grouping phenomenon) were to happen, crowding could fail and subjects would become aware of the adaptation direction for the remainder of the experimental session.

\section{Method}

The methods in this experiment were similar to those of Experiment 2A, except that the two pairs of adaptation Gabors did not contain motion in the same direction across trials. The direction of motion in each vertically aligned pair of Gabors was randomly determined on each trial. That is, although the top Gabor of the vertical pair always contained motion opposite the direction of the bottom Gabor, the motion of the top Gabor could be leftward or rightward on each trial. All other Gabors in the array contained leftward or rightward motion, determined randomly on each trial for each Gabor. Because the direction of motion in the test locations was randomized on each trial, there was no consistent direction of adaptation across trials. This would normally cause a saturation or adaptation within the test location to both directions of motion, which would serve to reduce the motion-induced shift found in the first experiment. To avoid this potential problem, fewer trials were presented (data were collected for only one representative condition), the "top-up" adaptation period was extended to $8 \mathrm{~s}$ (though the term "top-up" here is misleading, as the direction of motion on sequential trials could be in opposite directions), and, most important, the fixation point was repositioned at one of four different points on the screen on each trial (always $27^{\circ}$ from the screen center and a minimum of $21^{\circ}$ or $23^{\circ}$ from the nearest test Gabor for vertically and horizontally separated fixation points, respectively). The fixation point could be centered above, below, or to either side of the midpoint of the array of Gabors (from Figure 5; this reduced retinotopically specific saturation). On each trial, the fixation point jumped in a clockwise direction (e.g., if the fixation point was to the left of the array on Trial 1, it was above the array on Trial 2); this technique maximizes the interval between adaptation periods at each retinal location. Subjects fixated the bull's-eye at all times.

On the basis of the separation between each of the fixation points and the test Gabors, and the results of Experiment $1 \mathrm{C}$ above, subjects were unable to determine the direction of motion presented within either of the adaptation regions (Figure 7). The test Gabors had a fixed spatial frequency of 0.35 cycles/deg (identical to the low spatial frequency test Gabors in the first experiment), had an orientation orthogonal to the adaptation Gabors, and were presented for $240 \mathrm{~ms}$. There was no initial adaptation period. The procedure and method were otherwise identical to those of the first experiment.

\section{Results}

Figure 8 shows that there was still a perceived misalignment between the test Gabors even though the direction of motion adaptation was randomly chosen on each trial: for subject D.W., $t(30)=5.3, p<.05$; for subject D.B., $t(30)=8.6, p<.05$. The fact that there is a strong shift in the perceived position, even after only $8 \mathrm{~s}$ of motion adaptation, shows that brief exposure to motion is sufficient to bias subsequent position judgments. The results here also support Experiment 2A, showing that awareness (or becoming aware) of directional motion adaptation is not necessary for the effect.

\section{Experiment 2E: Correlating Crowding Effectiveness and Position Shifts}

Experiment 2C demonstrated that subjects were at chance when guessing the direction of motion in the test Gabors. Experiment 2D showed that there is a position shift even when the direction of motion in the test Gabors is randomized from trial to trial. To confirm that there is no correlation between the awareness of motion direction (i.e., the effectiveness of crowding) and the perceived position shift, we conducted another experiment in which subjects made two sequential judgments on each trial. Subjects judged the direction of motion in the crowded adaptation Gabor and also judged the position of the test Gabor. 

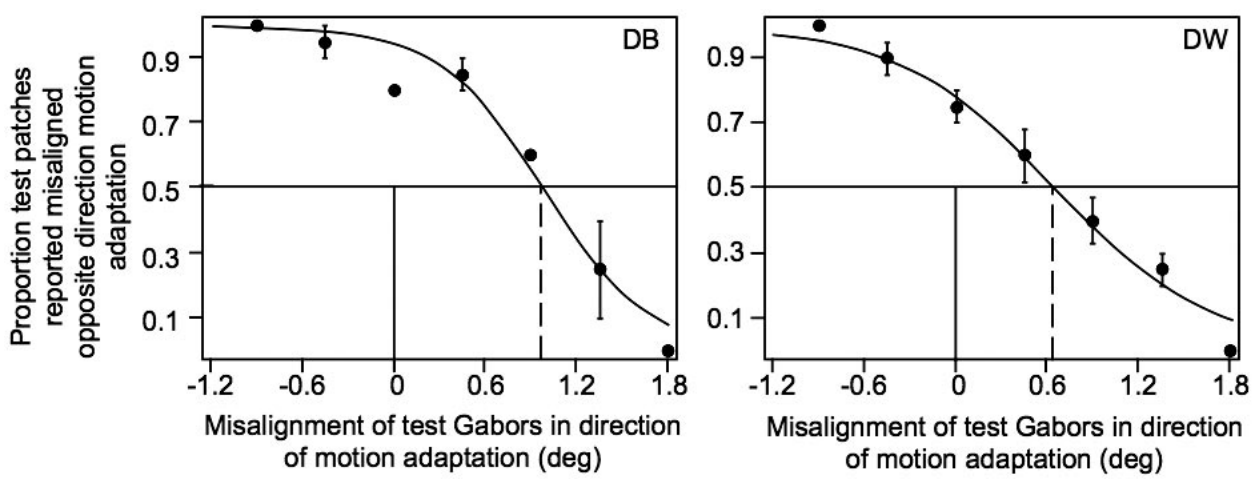

Figure 8. Results of Experiment 2D for 2 representative subjects. Psychometric functions revealed a strong shift in the perceived positions of the test Gabors, despite the fact that the direction of adaptation was randomly varied across trials (perceived misalignment was $1.0^{\circ}$ for D.B. and $0.62^{\circ}$ for D.W.). Error bars indicate $\pm 1 S E M$.

\section{Method}

The methods were similar to those in Experiment 2D. Two subjects participated in this experiment, both of whom had participated in the previous experiments (including the author). The fixation point remained in one position throughout the experiment (Figure 9). The direction of motion in each Gabor in the crowding array was randomly determined on every trial (spanning a range of $360^{\circ}$; Figure 9A). The adaptation Gabors (dashed circles) contained leftward or rightward drifting carriers, determined randomly on each trial. There was only one test location (dashed circles), which was always known to the subjects. There was no initial adaptation period. Each adaptation period (top-up adaptation) was $8 \mathrm{~s}$. The task was a combination of those in Experiments 2C and 2D: Subjects first judged the direction of motion in the upper adaptation Gabor (top dashed circle in Figure 9A). Subsequently, subjects judged the relative misalignment between the static test Gabors. The static test Gabors were identical to those in Experiment 2D (orthogonal to the adapting Gabors, as shown in Figure 5B). Subjects were allowed to make their first judgment at any moment during the trial but were encouraged to be as accurate as possible. Direction discrimination always preceded the judgment of Gabor position to avoid the possibility that the test Gabor might give away some information about the direction of motion adaptation. The locations of the test Gabors, the timing, the procedure, and the analysis were identical to those in Experiment 2D. Each subject participated in five sessions of 30 trials, for a total of 150 trials. A psychometric function was fit to the data using the methods described in Experiment 2A. Each session was separated by at least $30 \mathrm{~min}$ to reduce accumulated motion adaptation.

In the second half of the experiment, the same stimuli the above were used in a bias-free 2AFC task (MacMillan \& Creelman, 2004) to measure each subject's ability to discriminate direction of motion. The stimulus in Figure 9A was presented, and subjects were required to judge whether the upper or lower adaptation Gabor (upper and lower dashed circles in Figure 9A) contained rightward motion (a 2AFC task). Both leftward and rightward motion were always present, and the response (upper or lower) was therefore orthogonal to the presence of the stimulus (leftward or rightward motion), ensuring that the task was a bias-free estimate of discriminability (MacMillan \& Creelman, 2004; McKee et al., 1985). Calculating $d^{\prime}=$ $[z(H)-z(F)]$, where $H$ is the hit rate and $F$ is the false alarm rate, gives an unbiased estimate of discrimination (Green \& Swets, 1988; MacMillan \& Creelman, 2004).

\section{Results}

Figure 9B shows the results of Experiment 2E, revealing that subjects perceived a strong misalignment between the static test
Gabors while simultaneously being unable to report the direction of motion in the adaptation region. The smallest misalignment $\left(0.52^{\circ}\right)$ was for subject D.W. and was significant, $t(25)=4.41$, $p<.01$. The effect size was slightly smaller than that found in Figure 6 because the direction of adaptation could reverse from trial to trial. Subject D.W. reported the direction of motion at $52.6 \%$ accuracy, consistent with the results of Experiment 2C; if this observer were ever truly aware of motion direction, it must have been in some fraction of these $\sim 53 \%$ accurate responses. To ensure that these correct responses did not influence or determine the misalignment in Figure 9B, all of the correct responses were removed from the analysis and the psychometric function was recalculated (Figure 9B, dashed lines). Even without the accurate responses, both subjects still perceived a strong misalignment between the Gabors; the least significant effect was for subject D.W., $t(25)=3.35, p<.01$. There was not a significant difference between the perceived misalignment for accurate trials (hits) versus inaccurate trials (misses) for either subject: for subject D.W., $t(25)=0.92, p>.05$. In fact, for subject D.B., there was a slightly stronger position shift observed in the inaccurate trials (the dashed line is slightly to the right of the solid one in Figure 9B). These results provide strong evidence that the direction of motion adaptation was prevented and that there was nevertheless a shift in the perceived position of the test Gabors.

To further ensure that subjects were not able to discriminate the direction of motion in the adaptation Gabors (dashed circled regions in Figure 9A), we conducted another 2AFC experiment requiring subjects to discriminate the direction of motion in the top and bottom adaptation Gabors. Using an unbiased 2AFC task (MacMillan \& Creelman, 2004; McKee et al., 1985), $d^{\prime}$ was found to be 0.26 and 0.32 for subjects D.B. and D.W., respectively. This level of discrimination is not significant; the most significant effect was for subject D.W., $\chi^{2}(1,150)=0.15, p=.70$. Not surprisingly, without the added array of crowding Gabors (when just the two adaptation Gabors in the dashed circled regions were presented), discrimination was nearly perfect $\left(d^{\prime}>5\right)$. The crowded array of Gabors therefore eliminates observers' abilities to discriminate motion direction.

\section{Discussion: Experiments 2A-2E}

In the second experiment, we found that even when subjects could not distinguish the direction of motion in the adaptation 
A

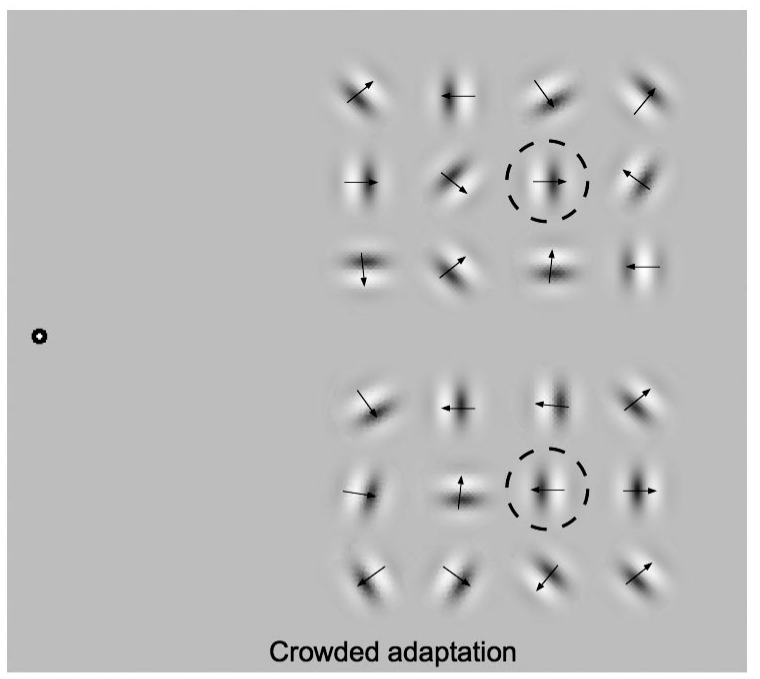

B
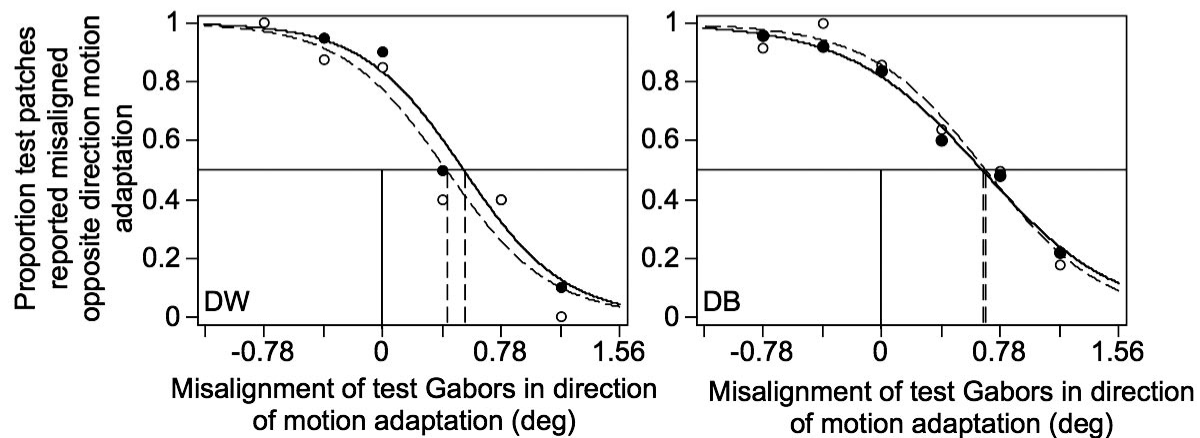

Figure 9. Stimulus and results of Experiment 2E for 2 subjects. A: Subjects adapted to a crowded array of Gabors drifting in random directions. In each trial, subjects judged the direction of motion in the adaptation Gabors (dashed circles, which were not visible in the experiment) and subsequently judged the relative alignment between the two static test Gabors (as in Experiment 2A). The direction of motion in the adaptation regions (dashed circles) was leftward or rightward, determined randomly on each trial. B: Psychometric functions for 2 subjects showing the perceived misalignment (shift) in the positions of the test Gabors (same format as Figure 8). The solid line is the psychometric function fit to all trials in the experiment; the least significant point of subjective equality (PSE) was for D.W.: $t(25)=3.35, p<.01$. The magnitude of this perceived position shift was consistent with Experiments $2 \mathrm{~A}$ and $2 \mathrm{D}$. The dashed line is the psychometric function fit only to the trials in which subjects incorrectly reported the direction of motion in the adaptation region (the top dashed circled region in $[\mathrm{A}])$. The PSEs of the psychometric functions were equivalent for hit and miss trials, revealing that the perceived shift in position did not correlate with accurate direction discrimination; the most significant difference between PSEs for hit and miss trials was for D.W.: $t(25)=0.92, p>.05$. In this experiment, accuracy at reporting direction of motion was .52 and .58 for subjects D.W. and D.B., respectively. In a separate, bias-free two-alternative forced-choice judgment (MacMillan \& Creelman, 2004), the discrimination of motion direction was found to be 0.26 and 0.32 ( $d^{\prime}$ units) for subjects D.B. and D.W., respectively. These $d^{\prime}$ values are at chance level; the best discrimination was for subject D.W.: $\chi^{2}(1)=0.15, p=.70$.

Gabors, the perceived position of subsequently viewed stimuli appeared shifted in position. This experiment contrasts with the first one and demonstrates that although high-level motion (motion driven by top-down processes) is sufficient to influence perceived position, there are also low-level passive motion detection mechanisms (operating in the absence of attentional scrutiny) that shift coded position. This experiment places at least some of the motion-induced position displacements (Whitney, 2002) at a stage prior to that at which crowding is determined. Regardless of what mechanism produces crowding (Bouma, 1973; Chung, Levi, \&
Legge, 2001; Field, Hayes, \& Hess, 1993; He et al., 1996; Intriligator \& Cavanagh, 2001; Kim \& Blake, 2005; Latham \& Whitaker, 1996; Levi et al., 1985; Pelli et al., 2004; Toet \& Levi, 1992) or where this occurs in the visual system, the results clearly show that motion and position interact prior to or in the absence of mechanisms requiring awareness of motion direction.

Recent experiments have shown that subjects can extract ensemble pattern information from crowded displays (Parkes, Lund, Angelucci, Solomon, \& Morgan, 2001). For example, the perceived global orientation of a crowded pattern is influenced by the 
orientation of a single central Gabor, even without awareness of that Gabor's orientation. In our experiment, it is unlikely that the perceived shift in the test Gabors was due to an extraction of ensemble statistics from the display as a whole, for several reasons. First, only the two aligned pairs of Gabor patterns at the center of the array had a consistent direction of motion across trials. All other Gabor motion was random and would prevent adaptation. Second, the averaged global motion of all Gabors was zero because the direction of motion was random for each Gabor on each trial; the adapted Gabors contained balanced motion that also had a net velocity of zero. Third, the perceived shift in the position of the test Gabors was highly position specific: The misalignment perceived between the test Gabors was different depending on the adapted region in which they were located, indicating that the perceived misalignment was not based on any spatial integration or ensemble statistic.

Additional alternative explanations for the results in this experiment can be ruled out. In addition to being spatially balanced and unpredictable, the local motion of each of the crowding Gabors in the array (those surrounding the adaptation Gabors) was balanced over time as well, because the direction of their motion was random on each trial. The misalignment is therefore due to prior exposure to local motion in the adaptation Gabors.

Prolonged exposure to motion in one location can influence the perceived motion (Anstis \& Reinhardt-Rutland, 1976; Ashida, Susami, \& Osaka, 1996; Culham et al., 2000; Snowden \& Milne, 1997; von Grünau \& Dube, 1992; Whitney \& Cavanagh, 2003) and positions (Whitney \& Cavanagh, 2003) of objects that are separated by a substantial distance from the region of motion adaptation. However, in this experiment, the illusory position shift was dominated by local motion adaptation; the shift was always opposite the direction of motion in the adapted location, not the motion in adjacent motion regions. The spatial extent of the adapted region was relatively small, indicating that the region over which motion signals are averaged (e.g., spatial summation; Anderson \& Burr, 1991; Braddick, 1993; Britten \& Heuer, 1999; Fredericksen, Verstraten, \& van de Grind, 1994; Nakayama, 1985) must be smaller than the size of the individual adaptation Gabors $\left(0.74^{\circ}\right.$ standard deviation, $\sim 4.5^{\circ}$ center-to-center separation between each Gabor). Several previously reported estimates of perceptive field size (i.e., the area of spatial summation or pooling) are consistent with the estimate here (Anstis \& Harris, 1987; Thorson et al., 1969). In addition to revealing a relatively small perceptive field, the local nature of the effect here may mean that although perceived position depends on the prior motion in a scene, there is a stronger weight assigned to the motion in the same location as the test object.

\section{General Discussion}

The experiments reported here demonstrate independent contributions of both top-down and bottom-up motion processes to perceived position. Using transformational apparent motion, an illusory motion in which there is no physical movement in the scene, we found that perceived position of nearby static objects depended on the direction of the illusory motion. In the second set of experiments, we presented drifting patterns in a crowded display. Although subjects could not identify the direction of motion in the patterns, the perceived positions of subsequently viewed objects were shifted. Therefore, although high-level motion perception can influence the perceived locations of static objects, a high-level mechanism (e.g., attentional tracking, inferred motion, or some other top-down process) is not necessary to shift perceived position. Low-level motion, in the absence of top-down processes, independently influences perceived position. Together, these results indicate that the visual system uses both high- and low-level motion to code the locations of objects.

\section{Mechanisms of Motion Perception}

Both bottom-up (Adelson \& Bergen, 1985; Burr, Ross, \& Morrone, 1986; Zanker, 1997) and top-down (Cavanagh, 1992; Lu \& Sperling, 1995) mechanisms mediate motion perception. Both types of mechanisms are capable of coding motion and position. Top-down mechanisms must explicitly represent the positions of objects or features in order to track them (Cavanagh, 1992; Lu \& Sperling, 1995). Bottom-up, spatiotemporal receptive fields tuned to velocity are able to simultaneously represent object form and motion (Burr et al., 1986; Nishida, 2004). Support for these bottom-up mechanisms includes a diverse array of illusions, including motion interpolation - the illusory perception of an apparently moving object occupying intermediate positions between its actually displaced positions (Burr, 1979; Fahle \& Poggio, 1981; Morgan, 1976; Shaw \& Ramachandran, 1982). Additional support is provided by the perception of speed lines (Burr \& Ross, 2002 Geisler, 1999) and the perception of form through slits (Nishida, 2004). This type of bottom-up motion detector could help explain some of the position displacements observed in moving objects. Additional nonlinear gain control mechanisms (Berry et al., 1999) or asymmetric connections created by spike-timing-dependent plasticity (Fu et al., 2001, 2004) could also shift the coded location of objects in the direction of motion. However, none of these bottom-up models predict that an illusory motion in which there is no physical or net motion signal in the scene (such as transformational apparent motion) should influence the perceived position of a static object-especially one that is separated from the motion by several degrees. Experiment 1, however, confirmed such a case.

\section{Time Course of Motion's Influence on Perceived Position}

It is unknown how or where perceived position is determined in the visual system. Multiple visual areas maintain retinotopic organization, and any of these areas could be necessary or sufficient for the perception of position. The experiments here suggest that there are multiple inputs of motion — both high and low level—-to perceived position.

Experiments $1 \mathrm{~B}$ and $1 \mathrm{C}$ revealed the time course of top-down motion's influence on perceived position. The broad temporal tuning of this effect (Figures 3 and 4) is consistent with previous studies that used first-order motion (Whitney \& Cavanagh, 2000). It remains to be determined whether the time course of motion's influence on position assignment is comparable for both bottom-up and top-down motion. Addressing this will help answer the question of whether the perceived location of an object is determined by a single neural mechanism or whether there are multiple independent position-coding mechanisms that may each contribute to perceived location. Motion-influenced position coding has been reported in the retina (Berry et al., 1999), as well as the primary 
visual cortex (Fu et al., 2004; Whitney et al., 2003), but all of these studies used first-order luminance-defined motion, presumably detected by passive mechanisms. The results presented here demonstrate that top-down motion influences perceived locations as well. Although high-level motion (e.g., Hikosaka et al., 1996; von Grünau et al., 1996; but cf. Downing \& Treisman, 1997; Zanker, 1997) may be processed in the primary visual cortex (V1) (Jancke, Chavane, Naaman, \& Grinvald, 2004), and attention modulates V1 activity (Somers, Dale, Seiffert, \& Tootell, 1999; Tootell et al., 1998; T. Watanabe et al., 1998), it remains to be seen whether this sort of top-down motion influences position coding in early visual areas.

\section{References}

Adelson, E. H., \& Bergen, J. R. (1985). Spatiotemporal energy models for the perception of motion. Journal of the Optical Society of America A, 2, 284-299.

Aghdaee, S. M. (2005). Adaptation to spiral motion in crowding condition. Perception, 34, 155-162.

Aghdaee, S. M., \& Zandvakili, A. (2005). Adaptation to spiral motion: Global but not local motion detectors are modulated by attention. Vision Research, 45, 1099-1105.

Anderson, S. J., \& Burr, D. C. (1991). Spatial summation properties of directionally selective mechanisms in human vision. Journal of the Optical Society of America A, 8, 1330-1339.

Anstis, S., \& Harris, J. (1987). Magnification factor for adaptation of a visual transient mechanism. Journal of the Optical Society of America A, 4, 1688-1698.

Anstis, S. M., \& Reinhardt-Rutland, A. H. (1976). Interactions between motion aftereffects and induced movement. Vision Research, 16, 13911394.

Ashida, H., Susami, K., \& Osaka, N. (1996). Re-evaluation of local adaptation for motion aftereffect. Perception, 25, 1065-1072.

Assad, J. A., \& Maunsell, J. H. (1995, February 9). Neuronal correlates of inferred motion in primate posterior parietal cortex. Nature, 373, $518-$ 521

Baldo, M. V., \& Klein, S. A. (1995, December 7). Extrapolation or attention shift? Nature, 378, 565-566.

Baloch, A. A., \& Grossberg, S. (1997). A neural model of high-level motion processing: Line motion and formotion dynamics. Vision Research, 37, 3037-3059.

Berry, M. J., II, Brivanlou, I. H., Jordan, T. A., \& Meister, M. (1999, March 25). Anticipation of moving stimuli by the retina. Nature, 398, 334-338.

Bouma, H. (1970, April 11). Interaction effects in parafoveal letter recognition. Nature, 226, 177-178.

Bouma, H. (1973). Visual interference in the parafoveal recognition of initial and final letters of words. Vision Research, 13, 767-782.

Braddick, O. (1993). Segmentation versus integration in visual motion processing. Trends in Neurosciences, 16, 263-268.

Britten, K. H., \& Heuer, H. W. (1999). Spatial summation in the receptive fields of MT neurons. Journal of Neuroscience, 19, 5074-5084.

Burr, D. C. (1979). Acuity for apparent vernier offset. Vision Research, 19, 835-837.

Burr, D. C., \& Ross, J. (2002). Direct evidence that "speedlines" influence motion mechanisms. Journal of Neuroscience, 22, 8661-8664.

Burr, D. C., Ross, J., \& Morrone, M. C. (1986). Seeing objects in motion. Proceedings of the Royal Society of London, Series B, 227, 249-265.

Cavanagh, P. (1992, September 11). Attention-based motion perception. Science, 257, 1563-1565.

Chatterjee, S. H., Freyd, J. J., \& Shiffrar, M. (1996). Configural processing in the perception of apparent biological motion. Journal of Experimental Psychology: Human Perception and Performance, 22, 916-929.
Chaudhuri, A. (1990, March 1). Modulation of the motion aftereffect by selective attention. Nature, 344, 60-62.

Chung, S. T., Levi, D. M., \& Legge, G. E. (2001). Spatial-frequency and contrast properties of crowding. Vision Research, 41, 1833-1850.

Cropper, S. J., \& Derrington, A. M. (1996, January 4). Rapid colourspecific detection of motion in human vision. Nature, 379, 72-74.

Culham, J. C., Verstraten, F. A., Ashida, H., \& Cavanagh, P. (2000). Independent aftereffects of attention and motion. Neuron, 28, 607-615.

De Valois, R. L., \& De Valois, K. K. (1991). Vernier acuity with stationary moving Gabors. Vision Research, 31, 1619-1626.

Downing, P. E., \& Treisman, A. M. (1997). The line-motion illusion: Attention or impletion? Journal of Experimental Psychology: Human Perception and Performance, 23, 768-779.

Duncker, K. (1938). Induced motion. In W. D. Ellis (Ed.), A source book of Gestalt psychology (pp. 161-172). London: Routledge \& Kegan Paul. (Original work published 1929)

Durant, S., \& Johnston, A. (2004). Temporal dependence of local motion induced shifts in perceived position. Vision Research, 44, 357-366.

Edwards, M., \& Badcock, D. R. (2003). Motion distorts perceived depth. Vision Research, 43, 1799-1804.

Fahle, M., \& Poggio, T. (1981). Visual hyperacuity: Spatiotemporal interpolation in human vision. Proceedings of the Royal Society of London, Series B, 213, 451-477.

Fang, F., \& He, S. (2004). Strong influence of test patterns on the perception of motion aftereffect and position. Journal of Vision, 4, $637-642$.

Farrell, J. E., \& Shepard, R. N. (1981). Shape, orientation, and apparent rotational motion. Journal of Experimental Psychology: Human Perception and Performance, 7, 477-486.

Felisberti, F. M., Solomon, J. A., \& Morgan, M. J. (2005). The role of target salience in crowding. Perception, 34, 823-833.

Festa, E. K., \& Welch, L. (1997). Recruitment mechanisms in speed and fine-direction discrimination tasks. Vision Research, 37, 3129-3143.

Field, D. J., Hayes, A., \& Hess, R. F. (1993). Contour integration by the human visual system: Evidence for a local "association field." Vision Research, 33, 173-193.

Finney, D. J. (1971). Probit analysis (3rd ed.). Cambridge, England: Cambridge University Press.

Fredericksen, R. E., Verstraten, F. A. J., \& van de Grind, W. A. (1994). Spatial summation and its interaction with the temporal integration mechanism in human motion perception. Vision Research, 34, 31713188.

Fu, Y. X., Shen, Y., \& Dan, Y. (2001). Motion-induced perceptual extrapolation of blurred visual targets. Journal of Neuroscience, 21, RC172.

Fu, Y. X., Shen, Y., Gao, H., \& Dan, Y. (2004). Asymmetry in visual cortical circuits underlying motion-induced perceptual mislocalization. Journal of Neuroscience, 24, 2165-2171.

Geisler, W. S. (1999, July 1). Motion streaks provide a spatial code for motion direction. Nature, 400, 65-69.

Georgeson, M. A., Freeman, T. C., \& Scott-Samuel, N. E. (1996). Subpixel accuracy: Psychophysical validation of an algorithm for fine positioning and movement of dots on visual displays. Vision Research, 36, 605-612.

Green, D. M., \& Swets, J. A. (1988). Signal detection theory and psychophysics (Rev. ed.). Los Altos, CA: Peninsula Publishing.

He, S., Cavanagh, P., \& Intriligator, J. (1996, September 26). Attentional resolution and the locus of visual awareness. Nature, 383, 334-337.

He, S., Cavanagh, P., \& Intriligator, J. (1997). Attentional resolution. Trends in Cognitive Sciences, 1, 115-121.

Hikosaka, O., Miyauchi, S., \& Shimojo, S. (1993). Focal visual attention produces illusory temporal order and motion sensation. Vision Research, $33,1219-1240$.

Hikosaka, O., Miyauchi, S., \& Shimojo, S. (1996). Orienting a spatial 
attention: Its reflexive, compensatory, and voluntary mechanisms. Brain Research: Cognitive Brain Research, 5(1-2), 1-9.

Intriligator, J., \& Cavanagh, P. (2001). The spatial resolution of visual attention. Cognitive Psychology, 43, 171-216.

Jancke, D., Chavane, F., Naaman, S., \& Grinvald, A. (2004, March 25). Imaging cortical correlates of illusion in early visual cortex. Nature, 428 , 423-426.

Kim, C. Y., \& Blake, R. (2005). Psychophysical magic: Rendering the visible "invisible." Trends in Cognitive Science, 9, 381-388.

Kooi, F. L., Toet, A., Tripathy, S. P., \& Levi, D. M. (1994). The effect of similarity and duration on spatial interaction in peripheral vision. Spatial Vision, 8, 255-279.

Krekelberg, B., \& Lappe, M. (2001). Neuronal latencies and the position of moving objects. Trends in Neurosciences, 24, 335-339.

Latham, K., \& Whitaker, D. (1996). Relative roles of resolution and spatial interference in foveal and peripheral vision. Ophthalmic and Physiological Optics, 16, 49-57

Levi, D. M., Klein, S. A., \& Aitsebaomo, A. P. (1985). Vernier acuity, crowding and cortical magnification. Vision Research, 25, 963-977.

Lu, Z. L., \& Sperling, G. (1995, September 21). Attention-generated apparent motion. Nature, 377, 237-239.

MacMillan, N. A., \& Creelman, C. D. (2004). Detection theory: A user's guide (2nd ed.). Mahwah, NJ: Erlbaum.

Mather, G., \& Morgan, M. (1986). Irradiation: Implications for theories of edge localization. Vision Research, 26, 1007-1015.

Mather, G., Verstraten, F., \& Anstis, S. (1998). The motion aftereffect: A modern perspective. Cambridge, MA: MIT Press.

McGraw, P. V., Walsh, V., \& Barrett, B. T. (2004). Motion-sensitive neurones in V5/MT modulate perceived spatial position. Current Biology, 14, 1090-1093.

McGraw, P. V., Whitaker, D., Skillen, J., \& Chung, S. T. (2002). Motion adaptation distorts perceived visual position. Current Biology, 12, 20422047.

McKee, S. P., Klein, S. A., \& Teller, D. Y. (1985). Statistical properties of forced-choice psychometric functions: Implications of probit analysis. Perception \& Psychophysics, 37, 286-298.

Morgan, M. J. (1976). Pulfrich effect and the filling in of apparent motion. Perception, 5, 187-195.

Morgan, M. J., Mather, G., Moulden, B., \& Watt, R. J. (1984). Intensityresponse nonlinearities and the theory of edge localization. Vision Research, 24, 713-719.

Mukai, I., \& Watanabe, T. (2001). Differential effect of attention to translation and expansion on motion aftereffects (MAE). Vision Research, 41, 1107-1117.

Mussap, A. J., \& Prins, N. (2002). On the perceived location of global motion. Vision Research, 42, 761-769.

Nakayama, K. (1985). Biological image motion processing: A review. Vision Research, 25, 625-660.

Nijhawan, R. (2002). Neural delays, visual motion and the flash-lag effect. Trends in Cognitive Sciences, 6, 387-393.

Nishida, S. (2004). Motion-based analysis of spatial patterns by the human visual system. Current Biology, 14, 830-839.

Nishida, S., \& Johnston, A. (1999, February 18). Influence of motion signals on the perceived position of spatial pattern. Nature, 397, 610612

Parkes, L., Lund, J., Angelucci, A., Solomon, J. A., \& Morgan, M. (2001). Compulsory averaging of crowded orientation signals in human vision. Nature Neuroscience, 4, 739-744.

Pelli, D. G., Palomares, M., \& Majaj, N. J. (2004). Crowding is unlike ordinary masking: Distinguishing feature integration from detection. Journal of Vision, 4, 1136-1169.

Pratt, J., \& Turk-Browne, N. B. (2003). The attentional repulsion effect in perception and action [Electronic version]. Experimental Brain Research, 152, 376-382.
Rajimehr, R., Montaser-Kouhsari, L., \& Afraz, S. R. (2003). Orientationselective adaptation to crowded illusory lines. Perception, 32, 11991210.

Rajimehr, R., Vaziri-Pashkam, M., Afraz, S. R., \& Esteky, H. (2004). Adaptation to apparent motion in crowding condition. Vision Research, 44, 925-931.

Ramachandran, V. S., \& Anstis, S. M. (1983, August 11). Perceptual organization in moving patterns. Nature, 304, 529-531.

Ramachandran, V. S., \& Anstis, S. M. (1990). Illusory displacement of equiluminous kinetic edges. Perception, 19, 611-616.

Ramachandran, V. S., Armel, C., Foster, C., \& Stoddard, R. (1998, October 29). Object recognition can drive motion perception. Nature, 395, 852 853.

Rock, I. (1990). The frame of reference. In I. Rock (Ed.), The legacy of Solomon Asch (pp. 243-268). Hillsdale, NJ: Erlbaum.

Roelofs, C. O. (1935). Die optische localisation [Optical localization]. Archiv für Augenheilkunde, 109, 395-415.

Seiffert, A. E., Somers, D. C., Dale, A. M., \& Tootell, R. B. (2003). Functional MRI studies of human visual motion perception: Texture, luminance, attention and after-effects. Cerebral Cortex, 13, 340-349.

Shaw, G. L., \& Ramachandran, V. S. (1982). Interpolation during apparent motion. Perception, 11, 491-494.

Shim, W. M., \& Cavanagh, P. (2004). The motion-induced position shift depends on the perceived direction of bistable quartet motion. Vision Research, 44, 2393-2401.

Snowden, R. J. (1998). Shifts in perceived position following adaptation to visual motion. Current Biology, 8, 1343-1345.

Snowden, R. J., \& Milne, A. B. (1997). Phantom motion after effects: Evidence of detectors for the analysis of optic flow. Current Biology, 7, 717-722.

Somers, D. C., Dale, A. M., Seiffert, A. E., \& Tootell, R. B. (1999). Functional MRI reveals spatially specific attentional modulation in human primary visual cortex. Proceedings of the National Academy of Science, USA, 96, 1663.

Stelmach, L. B., \& Herdman, C. M. (1991). Directed attention and perception of temporal order. Journal of Experimental Psychology: Human Perception and Performance, 17, 539-550.

Stuart, J. A., \& Burian, H. M. (1962). A study of separation difficulty: Its relationship to visual acuity in normal and amblyopic eyes. American Journal of Ophthalmology, 53, 471-477.

Suzuki, S. (2001). Attention-dependent brief adaptation to contour orientation: A high-level aftereffect for convexity? Vision Research, 41, 3883-3902.

Suzuki, S., \& Cavanagh, P. (1997). Focused attention distorts visual space: An attentional repulsion effect. Journal of Experimental Psychology: Human Perception and Performance, 23, 443-463.

Suzuki, S., \& Peterson, M. A. (2000). Multiplicative effects of intention on the perception of bistable apparent motion. Psychological Science, 11, 202-209.

Ternus, J. (1938). The problem of phenomenal identity. In W. D. Ellis (Ed.), A source book of Gestalt psychology (pp. 149-160). New York: Humanities Press.

Thornton, I. M. (2002). The onset repulsion effect. Spatial Vision, 15, 219-243.

Thornton, I. M., \& Hubbard, T. L. (2002). Representational momentum: New findings, new directions. London: Taylor \& Francis.

Thorson, J., Lange, G. D., \& Biederman-Thorson, M. (1969, May 30). Objective measure of the dynamics of a visual movement illusion. Science, 164, 1087-1088

Toet, A., \& Levi, D. M. (1992). The two-dimensional shape of spatial interaction zones in the parafovea. Vision Research, 32, 1349-1357.

Tootell, R. B., Hadjikhani, N., Hall, E. K., Marrett, S., Vanduffel, W., Vaughan, J. T., et al. (1998). The retinotopy of visual spatial attention. Neuron, 21, 1409-1422. 
Tse, P. U., \& Logothetis, N. K. (2002). The duration of 3-D form analysis in transformational apparent motion. Perception \& Psychophysics, 64, $244-265$.

von Grünau, M., \& Dube, S. (1992). Comparing local and remote motion aftereffects. Spatial Vision, 6, 303-314.

von Grünau, M., Dube, S., \& Kwas, M. (1996). Two contributions of motion induction: A preattentive effect and facilitation due to attentional capture. Vision Research, 36, 2447-2457.

Watanabe, K., Nijhawan, R., \& Shimojo, S. (2002). Shifts in perceived position of flashed stimuli by illusory object motion. Vision Research, 42, 2645-2650.

Watanabe, K., Sato, T. R., \& Shimojo, S. (2003). Perceived shifts of flashed stimuli by visible and invisible object motion. Perception, 32, $545-559$.

Watanabe, T., Harner, A. M., Miyauchi, S., Sasaki, Y., Nielsen, M., Palomo, D., et al. (1998). Task-dependent influences of attention on the activation of human primary visual cortex. Proceedings of the National Academy of Sciences, USA, 95, 11489-11492.

Whitaker, D., McGraw, P. V., \& Pearson, S. (1999). Non-veridical size perception of expanding and contracting objects. Vision Research, 39, 2999-3009.

Whitney, D. (2002). The influence of visual motion on perceived position. Trends in Cognitive Sciences, 6, 211-216.

Whitney, D. (2005). Motion distorts perceived position without awareness of motion. Current Biology, 15, R324-R326.
Whitney, D., \& Cavanagh, P. (2000). Motion distorts visual space: Shifting the perceived position of remote stationary objects. Nature Neuroscience, 3, 954-959.

Whitney, D., \& Cavanagh, P. (2003). Motion adaptation shifts apparent position without the motion aftereffect. Perception \& Psychophysics, 65, 1011-1018

Whitney, D., Goltz, H. C., Thomas, C. G., Gati, J. S., Menon, R. S., \& Goodale, M. A. (2003, October 31). Flexible retinotopy: Motiondependent position coding in the visual cortex [Electronic version]. Science, 302, 878-881.

Witkin, H., \& Asch, S. (1948). Studies in space orientation: IV. Further experiments on perception of the up-right with displaced visual fields. Journal of Experimental Psychology, 38, 762-782.

Wright, M. J., \& Johnston, A. (1985). Invariant tuning of motion aftereffect. Vision Research, 25, 1947-1955.

Zanker, J. M. (1997). Is facilitation responsible for the "motion induction" effect? Vision Research, 37, 1953-1959.

Zanker, J. M., Quenzer, T., \& Fahle, M. (2001). Perceptual deformation induced by visual motion. Naturwissenschaften, 88, 129-132.

Received April 30, 2005

Revision received January 23, 2006

Accepted January 23, 2006

\section{ORDER FORM}

Start my 2007 subscription to Journal of Experimental Psychology: Human Perception and Performance!

ISSN: 0096-1523

\section{\$148.00, APA Member/Affiliate \$316.00, INDIVIDUAL NONMEMBER $\$ 825.00$, InSTITUTION In DC add $5.75 \%$ / In MD add $5 \%$ sales tax} Total Amount Enclosed

\section{$\$$}

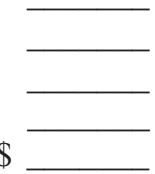

Subscription orders must be prepaid. (Subscriptions are on a calendar year basis only.) Allow 4-6 weeks for delivery of the first issue. Call for international subscription rates.

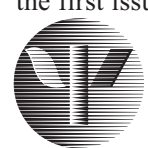

AMERICAN PSYCHOLOGICAL ASSOCIATION

\section{SEND THIS ORDER FORM TO:}

American Psychological Association Subscriptions 750 First Street, NE

Washington, DC 20002-4242

Or call 800-374-2721, fax 202-336-5568

TDD/TTY 202-336-6123

For subscription information, e-mail: subscriptions@apa.org
Check enclosed (make payable to APA)

Charge my: OVISA $\bigcirc$ MasterCard $\bigcirc$ American Express

Cardholder Name

Card No. Exp. Date

\section{BILLING ADDRESS:}

Street

City $\_$State __ Zip

Daytime Phone

E-mail

\section{MAIL TO:}

Name

Address

City State Zip

APA Member \# XHPA17 